\title{
A prototypical non-malignant epithelial model to study genome dynamics and concurrently monitor micro-RNAs and proteins in situ during oncogene-induced senescence
}

Eirini-Stavroula Komseli ${ }^{1 \dagger}$, loannis S. Pateras ${ }^{1 \dagger}$, Thorbjørn Krejsgaard ${ }^{2}$, Konrad Stawiski ${ }^{3}$, Sophia V. Rizou ${ }^{1}$, Alexander Polyzos ${ }^{4}$, Fani-Marlen Roumelioti ${ }^{4}$, Maria Chiourea ${ }^{4}$, loanna Mourkioti ${ }^{1}$, Eleni Paparouna ${ }^{1}$, Christos P. Zampetidis ${ }^{1}$, Sentiljana Gumeni ${ }^{5}$, loannis P. Trougakos ${ }^{5}$, Dafni-Eleftheria Pefani ${ }^{6}$, Eric O'Neill, Sarantis Gagos ${ }^{4}$, Aristides G. Eliopoulos ${ }^{7,8}$, Wojciech Fendler ${ }^{3,9}$, Dipanjan Chowdhury9, ${ }^{9,10}$, Jiri Bartek ${ }^{11,12,13^{*}}$ and Vassilis G. Gorgoulis ${ }^{1,4,14^{*}}$

\begin{abstract}
Background: Senescence is a fundamental biological process implicated in various pathologies, including cancer. Regarding carcinogenesis, senescence signifies, at least in its initial phases, an anti-tumor response that needs to be circumvented for cancer to progress. Micro-RNAs, a subclass of regulatory, non-coding RNAs, participate in senescence regulation. At the subcellular level micro-RNAs, similar to proteins, have been shown to traffic between organelles influencing cellular behavior. The differential function of micro-RNAs relative to their subcellular localization and their role in senescence biology raises concurrent in situ analysis of coding and non-coding gene products in senescent cells as a necessity. However, technical challenges have rendered in situ co-detection unfeasible until now.

Methods: In the present report we describe a methodology that bypasses these technical limitations achieving for the first time simultaneous detection of both a micro-RNA and a protein in the biological context of cellular senescence, utilizing the new commercially available SenTraGor ${ }^{T M}$ compound. The method was applied in a prototypical human non-malignant epithelial model of oncogene-induced senescence that we generated for the purposes of the study. For the characterization of this novel system, we applied a wide range of cellular and molecular techniques, as well as high-throughput analysis of the transcriptome and micro-RNAs.

(Continued on next page)
\end{abstract}

\footnotetext{
*Correspondence: jb@cancer.dk; vgorg@med.uoa.gr

${ }^{\dagger}$ Equal contributors

${ }^{11}$ Genome Integrity Unit, Danish Cancer Society Research Centre,

Strandboulevarden 49, DK-2100 Copenhagen, Denmark

${ }^{1}$ Molecular Carcinogenesis Group, Department of Histology and Embryology,

School of Medicine, National \& Kapodistrian University of Athens, 75 Mikras

Asias St, GR-11527 Athens, Greece

Full list of author information is available at the end of the article
} 
(Continued from previous page)

Results: This experimental setting has three advantages that are presented and discussed: i) it covers a "gap" in the molecular carcinogenesis field, as almost all corresponding in vitro models are fibroblast-based, even though the majority of neoplasms have epithelial origin, ii) it recapitulates the precancerous and cancerous phases of epithelial tumorigenesis within a short time frame under the light of natural selection and iii) it uses as an oncogenic signal, the replication licensing factor CDC6, implicated in both DNA replication and transcription when over-expressed, a characteristic that can be exploited to monitor RNA dynamics.

Conclusions: Consequently, we demonstrate that our model is optimal for studying the molecular basis of epithelial carcinogenesis shedding light on the tumor-initiating events. The latter may reveal novel molecular targets with clinical benefit. Besides, since this method can be incorporated in a wide range of low, medium or high-throughput imagebased approaches, we expect it to be broadly applicable.

Keywords: In situ hybridization, Micro-RNAs, Replication stress, Oncogene-induced senescence, CDC6, SenTraGorTM, DNA damage response, $\mathrm{R}$ loops, rDNA, Cancer

\section{Background}

For almost half a century RNA was considered just the coupler between DNA and protein production. On the other hand, proteins represented the main workhorse of the cell. This view has dramatically changed over the last years, as regulatory RNAs emerged as a versatile component of the molecular machinery interacting with both DNA and proteins "shaping" gene expression and protein function, respectively $[1,2]$. Mounting evidence shows that this bidirectional interplay plays a vital role in a variety of cellular responses, such as senescence; the latter involved in the pathogenesis of various diseases, including cancer.

Tumors are mixed tissues composed of cancer cells, with a tremendous phenotypic plasticity (tumor heterogeneity), and normal recruited cells that form the tumor-associated stroma [3, 4]. Under the microscope, a spatial organization of malignant and surrounding stromal cells is evident, implying a functional interplay between the various cell populations. Indeed, tumor development is mediated through a continuous "cross talk" between intra-cellular molecular pathways and intercellularly among cancer cells and the surrounding adjacent stroma [5]. The introduction of "-omics" increased our perception of the underlying processes occurring during carcinogenesis at a scale that has never been feasible before. However, the results of high-throughput analyses are in fact heterogeneous signals deriving from all the different cellular elements that comprise tumors. Thus, the molecular signature of distinct, relatively small tumor cell populations can be dramatically reduced or even lost, eliminating the ability to comprehend their contribution to cancer development. Hence, in situ detection of biomolecules rises as a crucial necessity to interrogate the mechanistic aspects of carcinogenesis.

In situ hybridization (ISH) and immune-localization assays are valuable tools for detecting spatial features of the transcriptional and translational machineries. ISH is among the most frequent techniques employed for the study of gene coding and non-coding RNAs (ncRNAs), including micro-RNAs (miRs). miRs have been demonstrated to play pivotal roles in multiple cell-fate decisions [6-12]. They are short, 22 nucleotides in length, highly conserved and, although abundant, escaped notice until 1993, when they were first reported by Lee, Freinbaum and Ambros [13]. Biogenesis of miRs begins with the generation of long primary transcripts, called primary miRs (pri-miRs), by RNA polymerase II (RNA pol II) (Additional file 1: Figure S1). Subsequently, pri-miRs are processed by Drosha, a class 2 RNase III, forming the precursor miRs (pre-miRs) that are exported in the cytoplasm, by exportin-5. In the cytoplasm Dicer, a class 3 RNase III, produces the mature single strand that is loaded on the RISC (RNA-induced silencing complex) effector complex [14, 15]. Pairing between miR and its target mRNA facilitated by Argonaute, a core component of RISC, promotes post-transcriptional down-regulation by cleavage and degradation (Additional file 1: Figure S1). Besides their cytoplasmic localization and role, a number of miRs have been discovered to function in the nucleus [16]. The picture becomes even more fascinating, as miRs have been detected in the mitochondria affecting their dynamics, further underscoring the importance of monitoring miRs spatial distribution $[17,18]$ (Additional file 1: Figure S1 and Additional file 2: Table S1).

Of particular interest is the role of miRs in cellular senescence [19-22] (Additional file 3: Table S2). The latter is a fundamental biological process involved in tissue homeostasis during normal development, and implicated in a broad spectrum of pathologies, including cancer [23-26]. In mammalian cells two types of cellular senescence have been recognized; namely, replicative senescence (RS) that occurs after certain number of cell divisions due to telomere attrition and stress-induced 
premature senescence (SIPS) [27]. SIPS is telomereindependent and represents an acute response to a wide range of stressful stimuli long before telomere erosion appears [27]. A decade ago, we and others demonstrated that oncogenes trigger SIPS and that this response constitutes a potent anti-tumor barrier [28-34]. This type of senescence is referred to as "oncogene-induced senescence" (OIS) [35] and according to the oncogene-induced DNA damage model for cancer development, OIS must be bypassed for tumor progression [36].

Even though senescence affects a variety of cell types, the in vitro cellular models recurrently used to study the phenomenon of OIS are almost always fibroblast-based (Additional file 4: Table S3). As most common malignancies are of epithelial origin, the lack of nonmalignant epithelial-derived models constitutes a major "gap" in the field. The basic grounds for the rareness of non-malignant epithelial-based cellular systems are: i) the difficulty in maintaining the specialized epithelial features during cultivation, rendering de-differentiation a frequent outcome [37], and ii) the unsuccessful immortalization with ectopic expression of human telomerase reverse transcriptase (hTERT) in epithelial cells in contrast to fibroblasts [38, 39].

Additionally, given the significance of miRs in senescence biology and their diverse intracellular localization (Additional file 1: Figure S1 and Additional file 2: Table S1) we were surprised to notice that there are no in situ studies of miRs in senescent cells (Additional file 3: Table S2). All the reports suffer from the limitation of analyzing miRs by qRT-PCR (quantitative reverse-transcription polymerase chain reaction) or micro-arrays of RNA isolated from cell cultures or tissues, independently scanned for senescence markers; thus lacking cellular specificity in cases of heterogeneity (Additional file 3: Table S2). The main reason behind the absence of in situ miR studies relies on restraints of the currently applied method for detecting senescence. The assay universally used, senescenceassociated $\beta$-galactosidase (SA- $\beta-\mathrm{Gal}$ ) assay, is based on the activity of $\beta$-galactosidase at suboptimal $\mathrm{pH}(\mathrm{pH}$ 6.0). Among the steps incorporated in this method are: i) instant application on fresh cells/tissues to avoid loss of enzymatic activity; thus rendering prior RNA detection unfeasible, and ii) a lengthy incubation of $12-16 \mathrm{~h}$ at $37^{\circ} \mathrm{C}$ in a complex ion buffer of $\mathrm{pH} 6.0$ containing magnesium ions $\left(\mathrm{Mg}^{+2}\right)$ [40], conditions which negatively affect RNA stability [41-45]. Although miRs are more stable than the larger RNA species [46-48], they eventually undergo degradation, as well $[49,50]$. As recently shown, in urine samples stored for prolonged time at room temperature their quantity decreases on average to $81 \%$ of their initial levels at 24-h intervals [51]. Another potential source of RNA degradation, during application of the aforementioned method, may be the presence of RNases that are known to be very stable enzymes and cannot be inactivated by the fixative procedure applied by Dimri et al. [40, 52]. As a result, prolonged incubations at $37{ }^{\circ} \mathrm{C}$ may allow the decay mechanisms to act in parallel exerting their activity.

We recently reported a universally applicable hybrid histo-/immunohisto-chemical method that can bypass all the aforementioned constraints of detection of senescent cells [53]. This is a non-enzymatic assay based on the property of a novel reagent, termed GL13, to detect lipofuscin, a non-degradable metabolic by-product that is considered a "hallmark" of senescence [54]. In the present study, we have developed a method that enables the simultaneous detection of gene coding (proteins) and non-coding (miRs) products in GL13-reactive senescent cells in situ. We have applied this methodology in an epithelial cell model that over-expresses the replication licensing factor CDC6 (Cell division cycle 6) in an inducible manner. We show that this cell system recapitulates the whole spectrum of epithelial carcinogenesis from the nonmalignant stage to oncogene-mediated activation of the anti-tumor barrier of senescence, followed by escape and the production of aggressive clones with features of epithelial to mesenchymal transition (EMT).

\section{Results}

The underlying principle for generating a CDC6-based non-malignant human epithelial model to study oncogene-induced senescence

According to a carcinogenesis model we have proposed, activated oncogenes disrupt normal DNA replication provoking replication stress that in turn triggers the DNA damage response (DDR) pathway, promoting genomic instability and cancer development [36]. An early feature of this process is that specific loci of the genome, called common fragile sites (CFS) are targeted resulting in breaks, gaps and rearrangements $[55,56]$. The carcinogenic process we describe is not an undemanding procedure as incipient cancer cells need to evade the anti-tumor barriers of apoptosis and senescence to evolve into full-blown malignant cells $[26,36]$. Thus, roughly the model can be divided into two phases: the first is typified by the activation of anti-tumor barriers, and the second is characterized by escape from the tumor-suppressor "blockade" driving cancer progression. The type of anti-tumor response elicited, apoptosis or senescence, is determined largely by the cellular context, however almost all studies, as already mentioned, examine the response to oncogene activation in mesenchymalbased cellular settings (Additional file 4: Table S3). Regarding the "escape from the tumor-suppressor barriers", the cancer biology field relies mainly on genetic manipulation of potential anti-tumor network players and monitoring of barrier circumvention. Although this experimental approach is useful in drawing conclusions 
about the role of a particular factor, it lacks the element of "natural evolution".

The requirement of a non-malignant model to perform an epithelial cancer evolution experiment (chronic expression of the oncogene without genetic intervention upon its activation) led us to utilize human bronchial epithelial cells (HBECs) as a platform to generate an oncogene doxycycline-inducible (Tet-ON) cellular system. Immortalization with combined expression of hTERT and ectopic mutant cyclin-dependent kinase 4 (CDK4) was successful in order to bypass $\mathrm{p} 16^{\mathrm{INK} 4 \mathrm{~A}}$-induced premature growth arrest (Additional file 5: Figure S2), maintaining the epithelial phenotype of the cells [57-59]. Further genetic manipulations to develop the inducible system also did not affect the epithelial characteristics of this model (Fig. 1a). We employed the replication licensing factor CDC6 as an inducible oncogene for the following reasons: $\mathbf{i}$ ) it is a key component of the replication licensing machinery, found to be frequently deregulated in cancer from its earliest stages [60-62], and ii) when over-produced it displays a multi-functional facet by compromising the replication process (re-replication: a form of replication stress) triggering genomic instability $[33,60,63,64]$, and acquiring properties of a transcriptional regulator affecting: a) negatively the expression of the nodal tumor-suppressors loci, INK4/ARF (encoding $\mathrm{p} 16^{\mathrm{INK} 4 \mathrm{~A}}, \mathrm{ARF}$ and $\mathrm{p} 15^{\mathrm{INK} 4 \mathrm{~B}}$ ) and $\mathrm{CDH1}$ (encoding E-cadherin) [62, 63, 65] (Additional file 6: Figure S3),

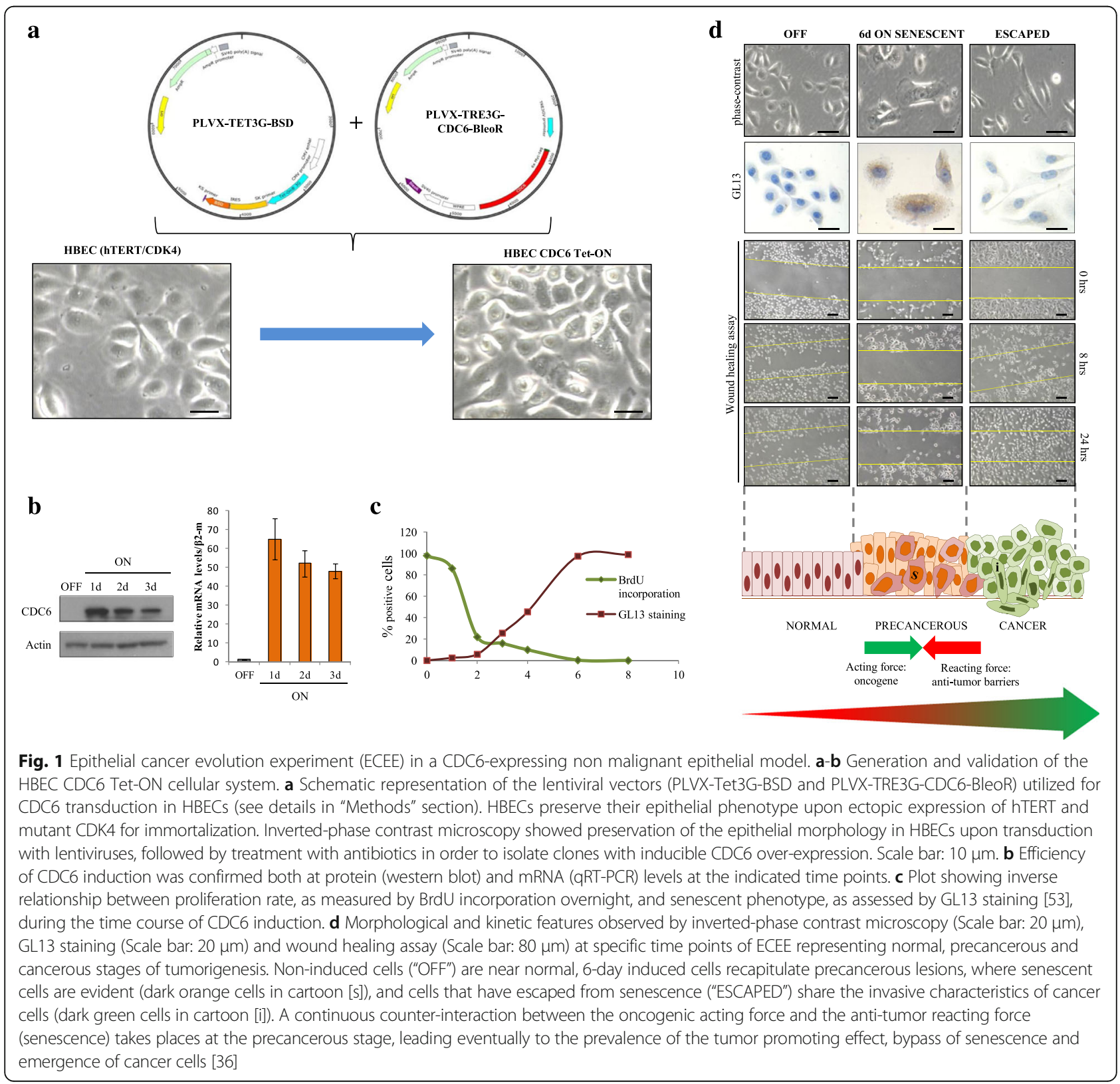


and b) positively that of $r D N A$ (ribosomal DNA), most probably impinging on RNA dynamics [66].

\section{Epithelial cancer evolution experiment (ECEE)}

Subsequent to validating the efficacy of CDC6 induction in the newly formed HBEC CDC6 Tet-ON system (Fig. 1b), we constitutively expressed it at levels relevant to those of tumor samples and monitored the cellular behavior over time. Stimulation of CDC6 resulted in a progressive decrease of cell proliferation, as depicted by reduced BrdU (bromodeoxyuridine) incorporation (Fig. 1c), that ceased after 6 days of induction. As proliferation diminished, the cells after a 3-day induction gradually acquired a senescent phenotype, as depicted by GL13 staining [53], that peaked at day 6 (Fig. 1c-d and Additional file 7: Figure S4). This finding is in line with a previous study where we showed that forced expression of CDC6 in human fibroblasts, induced senescence in a DDR-dependent manner [33]. Compared to fibroblasts, we were surprised by the robustness of this reaction in HBECs. This is quite intriguing given that senescence seems to be the predominant stress response program in fibroblasts, whereas apoptosis is believed to be the prevalent route in epithelial cells [67]. However, a recent report showing CDC6-mediated inhibition of apoptosome formation through its binding onto cytochrome $c$-activated Apaf-1 [68], probably explains why senescence emerges as the only tumor suppressor mechanism in our system. From a morphological point of view, microscopical analysis revealed enlarged and flattened cellular shapes, occasionaly multinucleated (Fig. 1d and Additional file 7: Figure S4), without the appearance of senescence associated heterochromatin foci (SAHF). Notably, this feature is observed during irreversible senescence in cells with an intact $\mathrm{p} 16^{\mathrm{INK} 4 \mathrm{~A}} / \mathrm{Rb}$ pathway, justifying its absence in our system [69]. On the other hand, the senescent cells presented extensive vacuolation, as well as the formation of extracellular vesicles indicative of increased secretoty activity, which may be related to the senescence associated secretory phenotype (SASP), the so called "dark side" of the senescent program accountable for its protumorigenic properties [26] (Fig. 2a). After a protracted stalled growth phase (around a month), when all cells were senescent and uniformaly expressed CDC6 (Figs. 1d and 2b-c and
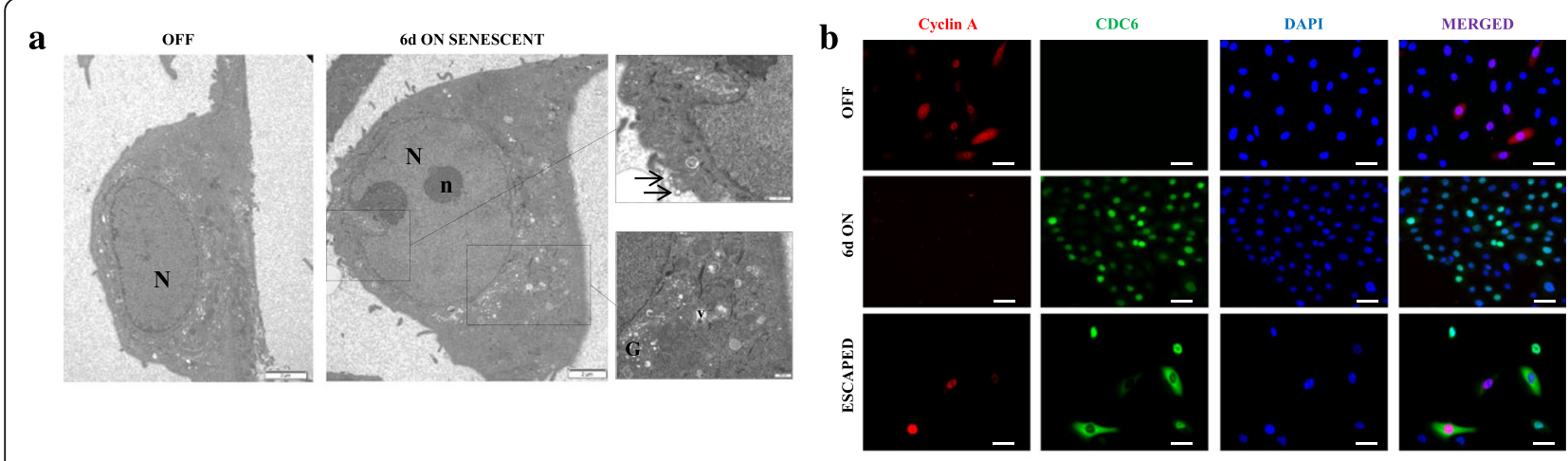

c

E-cadherin
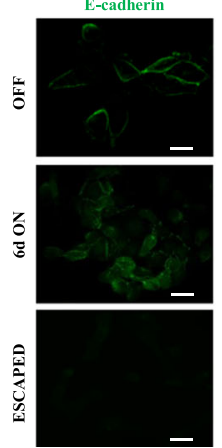

CDC

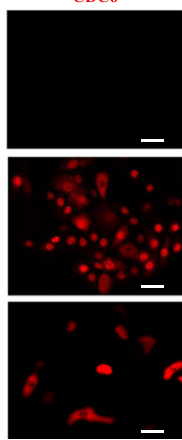

DAPI

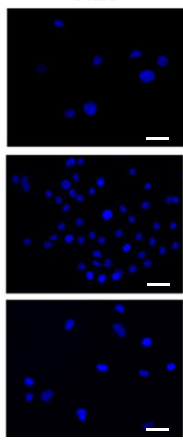

MERGED

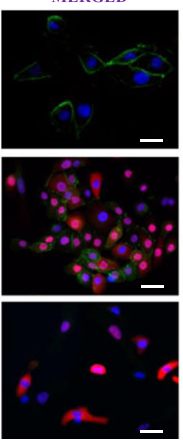

Vimentir
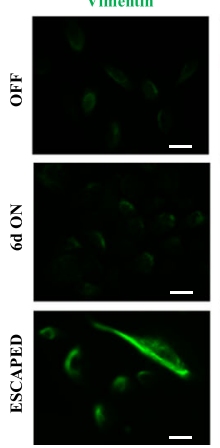

$\mathrm{CDC} 6$

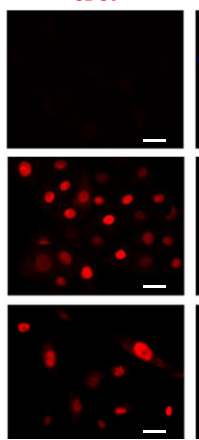

DAPI

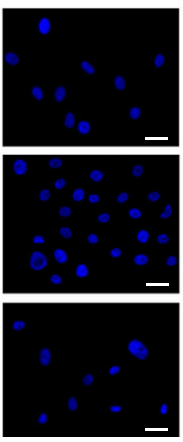

MERGED

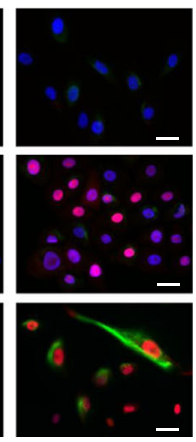

Fig. 2 Phenotypic characterization of HBEC CDC6 Tet-ON system during ECEE. a Ultrastructural analysis of 6 days induced cells revealed flattened cellular morphology, with enlarged nucleus (N), enhanced protein-synthesis, as visualized by double nucleoli (n) and extensive Golgi apparatus $(\mathrm{G})$, and formation of extracellular vesicles (v). Arrows depict extracellular vesicles. Scale bars $2 \mu \mathrm{m}$ and $500 \mathrm{~nm}$. b IF analysis of the proliferative marker (Cyclin A) and CDC6 showed double positive cells only after escape from senescence. Scale bar: $15 \mu \mathrm{m}$. c IF for an epithelial marker (E-cadherin) or a mesenchymal marker (Vimentin) along with CDC6 indicated EMT in the "escaped" cells. IF for CDC6 showed ubiquitous and uniform expression in the induced cells ("ON" and "ESCAPED"). Scale bar: $20 \mu \mathrm{m}$ 
Additional file 7: Figure S4), a fraction of proliferating cells emerged with distinct morphological features compared to those of the "OFF" state. These cells, from now on termed "escaped", showed traces of lipofuscin during the first cell divisions, proving that they came from senescent cells, while they were negative for GL13 after several passages and serial dilutions of the non-degradable metabolic by-product (Fig. 1d and Additional file 7: Figure S4). Moreover, they were double positive for CDC6 and Cyclin A, a well characterized cell proliferation marker (Fig. 2b). Unexpectedly, they attained a spindle morphology resembling that of mesenchymal cells (Fig. 1d and Additional file 7: Figure S4), insinuating an EMT, an embryonic program implicated in cancer invasion and progression [70, 71]. In accordance, E-cadherin, a fundamental adhesion molecule of epithelial tissues, was lost in the "escaped" cells, identifying a cardinal feature of the EMT program, whereas vimentin, a mesenchymal marker, increased (Fig. 2c) [71]. From a kinetic angle, carrying out a migration assay (wound healing assay) we observed that the "escaped" cells migrated significantly faster than the noninduced ones, whereas the senescent cells were completely still (Fig. 1d). To sum up, we developed an in vitro oncogene-regulated non-malignant human epithelial model that could compellingly recapitulate the precancerous and cancerous phases of the model we proposed (Fig. 1d) [36].

\section{Mechanistic insights into the senescent and escape phases of the ECEE}

To gain a mechanistic view underlying the phenotypic phases we described (Figs. 1-2 and Additional file 7: Figure S4) we followed a dual approach: i) based on our prior knowledge, we hypothesized and examined, whether senescence was DRR-induced [33, 36, 60, 72-74] and ii) we performed high-throughput analyses of transcriptome and miRs. We performed transcriptome analysis at two time points, one at day 3 (initiation of the senescent phase) and a second during the "escaped" phase (evolution of the protumorigenic phase) to unravel potential cancer initiating and driver events, respectively, while we added another time point at day 6 (full senescent phenotype) in order to reveal a potential miR signature of the senescent cells.

In line with our previous reports [33, 60, 75], CDC6 induction resulted in re-replication (cells with DNA content $>4 n$ ) (Fig. 3a), a form of replication stress, that leads to replication fork stalling, collapse, DNA damage and DDR activation. DNA damage was documented by alkaline comet assay (Fig. 3b), and DDR stimulation by 53BP1 foci formation and induction of the p53 pathway (Fig. 3c). The emergence of the "escaped" cells was distinguished, apart from the EMT features, by the attenuation of the DNA damage and the DDR pathway, implying that a repair process took place. A cytogenetic analysis of the "escaped" cells, relative to the non-induced

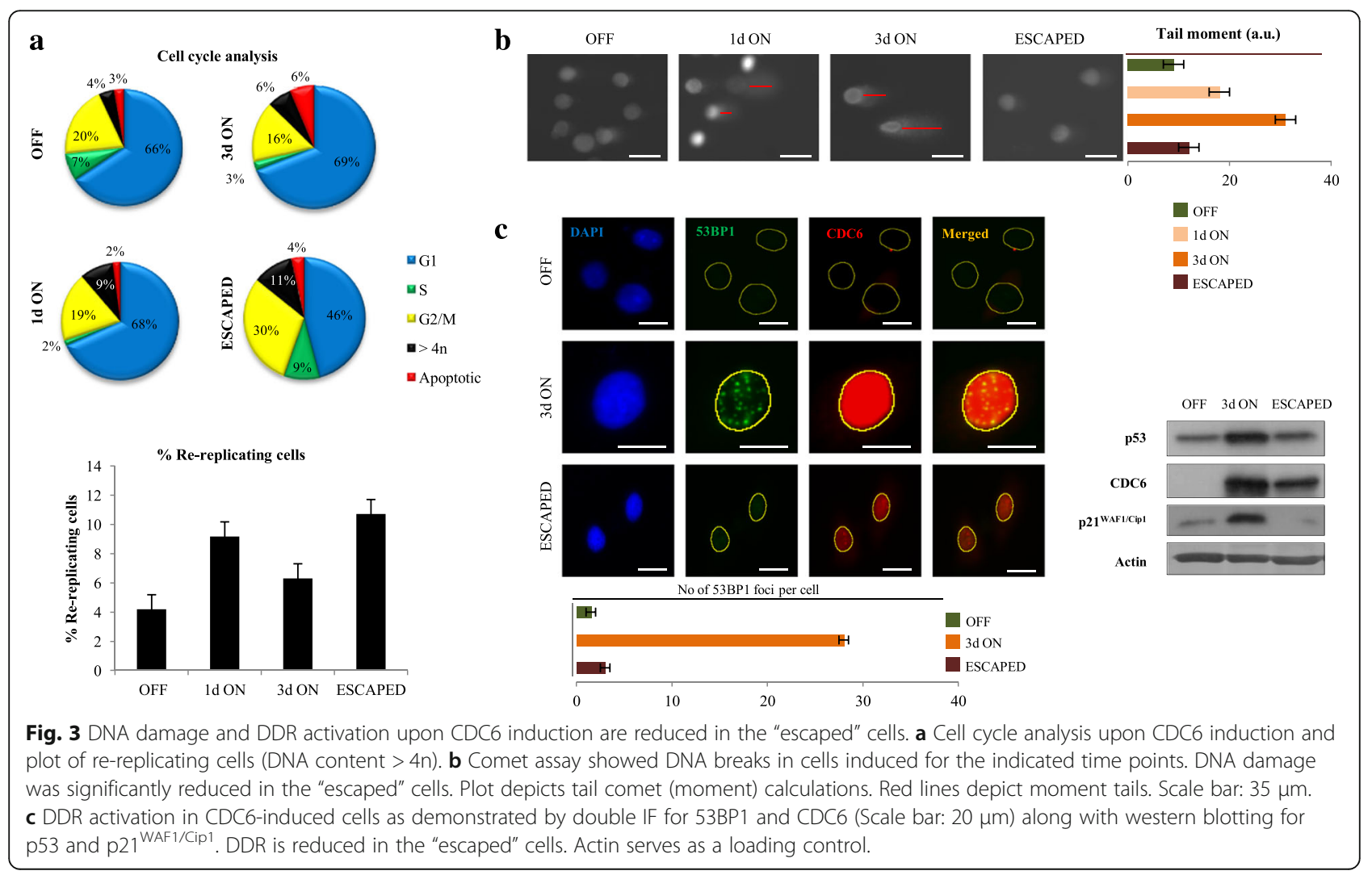




\section{HBEC CDC6 Tet-ON}

a

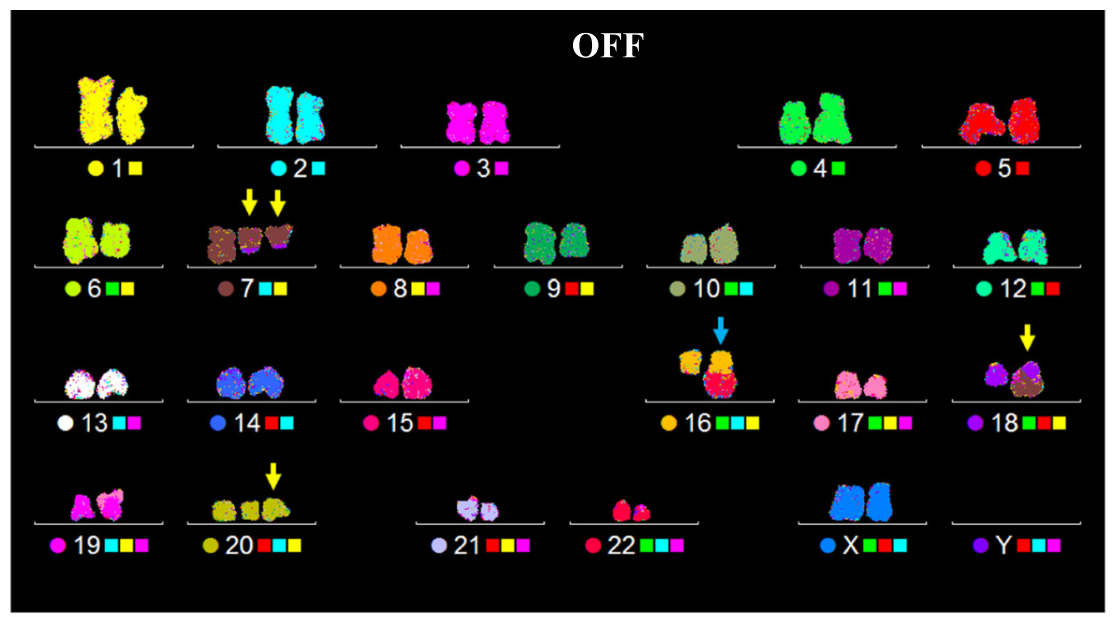

48,XX,der(7)t(7;18)(q11.2;q22.2),+der(7)t(7;18)(q11.2;q22.2),der(16)t(5;16)(q11.2;q24), $\operatorname{der}(18) t(7 ; 18)(q 11.2 ; q 22.2), \operatorname{der}(19) t(17 ; 19)(\mathbf{p 1 3 . 3} ; \mathbf{q} 23.1),+\operatorname{der}(20) \operatorname{dup}(q 13.13 q 13.33)$



48,XX,i(5p),+i(5p),der(6)t(6;9)(q23.3;q32),del(8)(p23.1), +der(9)i(9q?)ins(q32?;?), $+\operatorname{der}(12) t(1 ; 12)(p 11 ; q 24.1), \operatorname{der}(16) t(5 ; 16)(q 11.2 ; q 24), \operatorname{der}(20) t(16 ; 20)(q 22.2 ; q 13.1)$

c

\begin{tabular}{|c|c|}
\hline Break-point & Fragile site \\
\hline $1 \mathrm{p} 11$ & FRA1O \\
\hline $6 \mathrm{q} 23.3$ & FRA6L \\
\hline $8 \mathrm{p} 23.1$ & FRA8G \\
\hline $9 \mathrm{q} 32$ & FRA9B \\
\hline $12 \mathrm{q} 24.1$ & FRA12E \\
\hline $16 \mathrm{q} 22.2$ & FRA16D \\
\hline $20 \mathrm{q} 13.1$ & FRA20E \\
\hline
\end{tabular}

\section{b Clonal aberrations}

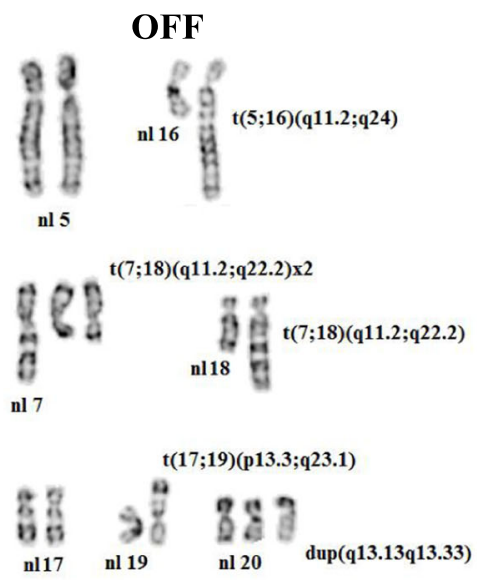

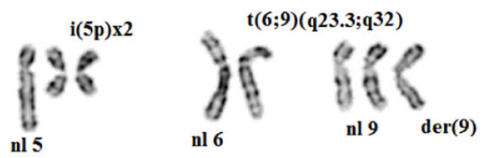

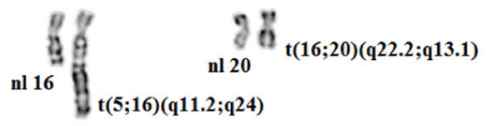




\section{(See figure on previous page.)}

Fig. 4 Cytogenetic analysis revealed an altered karyotype in the "escaped" cells, displaying novel clonal numerical and structural chromosome aberrations coinciding with common fragile sites (CFS). a Multi-Color FISH spectral karyotyping (M-FISH/SKY) of the non-induced and the "escaped" HBECs combined with inverted DAPI banding, as described in [143], revealed a near diploid numerical constitution of 48 chromosomes. Yellow arrows indicate unique clonal rearrangements for each population whereas the blue arrows depict the common structural anomaly der(16)t(5;16). $\mathbf{b}$ Partial karyotypes in Inverted DAPI Banding demonstrate the breakpoints of clonal structural chromosome rearrangements in the two populations, OFF (upper panel) and "escaped" (lower panel). nl: normal copy. Several clonal structural rearrangements characteristic of the control cells (OFF), were lost in the cytogenetically examined "escaped" cells; these included the products of the translocation t(7;18), der(7)t(7;18)(q11.2;q22.2)(found in two copies) and der(18)t(7;18)(q11.2;q22.2). Lost in the "escaped" cells were also the der(19)t(17;19)(p13.3;q23.1) and the extra copy of chromosome 20 bearing a duplication of the long arm. Despite the clonal chromosome losses, the "escaped" cells displayed at least 6 novel clonal structural chromosome anomalies and their cytogenetic constitution was shaped by non-disjunctions of chromosomes 1, 5, 7, 12 16, 18 and 19. The karyotype of the "escaped" cells was characterized by the clonal gain of genomic material from $1 q$ and most of chromosome 12, introduced by the emergence of the novel unbalanced translocation involving chromosomes 1 and 12, $\operatorname{der}(12) t(1 ; 12)(p 11 ; q 24.1)$, as well as deletions of 8p23.1. In addition to these novel anomalies, the "escaped" cells presented an unbalanced translocation involving chromosomes 6 and 9, an extra copy of a rearranged derivative of chromosome 9, a translocation between 16 and 20 and two copies of isochromosome 5p. c Breakpoints of all the novel structural chromosome rearrangements identified in the "escaped" HBECs coincide with common aphidicolin induced fragile sites [78]

(OFF) ones, revealed the presence of an altered karyotype, implying that this repair process was error-prone. The "escaped" cells harbored novel clonal (numerical and structural) chromosomal alterations and an increased rate of random structural chromosome rearrangements, indicative of genomic instability (Fig. 4a-b and Additional file 8: Figure S5) [76, 77]. Of note, we have observed a similar molecular response in cells that "escaped" from protracted p21 ${ }^{\mathrm{WAF} / \mathrm{Cip} 1}$-mediated senescence [64]. Remarkably, the majority of the novel breakpoints identified in the "escaped" HBECs coincided with aphidicolin-induced CFS (Fig. 4c) [78], a finding that is in line with the proposed oncogene-induced DNA damge model for cancer development [36]. Interestingly, the karyotype of the "escaped" cells was also characterized by gains of $1 q$ and $5 p$ and deletions of 8p23.1, which are frequently found in common solid cancers and various hematologic malignancies, and are associated with poor prognosis [79-82].

Even though replication stress via re-replication can explain CDC6-mediated genomic instability, a recent report showing that CDC6 could regulate $r D N A$ transcription initiation [66] led us to presume that an additional source of genomic instability could be R loop formation. $\mathrm{R}$ loops are three-stranded nucleic acid structures that encompass nascent RNA hybridized with DNA template, leaving single-stranded the non-template DNA (ssDNA) [83] (Additional file 9: Figure S6). Their formation and/or stabilization, which follows transcription, depends on various factors such as, high $\mathrm{G}$ density, negative supercoiling, DNA nicks and G-quartets in the displaced ssDNA $[84,85]$, and if persistent they set genome integrity at "risk" [83]. They are also frequently produced at CFS, regions of the genome prone to replication stress, located in long human genes $(\geq 800 \mathrm{~kb})$; thus increasing the possibility of replication-transcription collision and genomic instability [56]. The fact that $\mathrm{R}$ loops are reported in vivo at origins of replication [86-90] and rDNA loci [91, 92] increases the probability of their formation by deregulated expression of the replication licensing factor, CDC6.
To examine the above scenario we first measured total transcription levels by quantifying 5 '-ethynyl uridine (5'-EU) incorporation at specific time points, pre- and post-CDC6 induction. We observed a gradual increase of 5'-EU integration that peaked in the "escaped" cells (Fig. 5a). Re-replication and RNA production could well form a permissive genome landscape for the generation of $\mathrm{R}$ loops. Indeed, applying a specific antibody that detects DNA:RNA hybrids (S9.6) revealed a raise in $\mathrm{R}$ loop formation within the nucleoli in the initial phases of CDC6 expression that ceased during senescence, possibly due to recession of DNA replication, but reappeared in the "escaped" cells (Fig. 5b). The specificity of the reaction was verified by treating the cells with RNase A or RNase $\mathrm{H}$, enzymes specific in removing DNA:RNA hybrids [93, 94] (Fig. 5b). If $\mathrm{R}$ loops were related to DNA damage in our setting, then a repositioning of damaged rDNA on the nucleolar surface should occur facilitating recruitment of repair factors. This type of peripheral re-localization of the repair process is characteristic of heterochromatin enriched structures, such as the nucleoli $[95,96]$. In agreement with the latter, induction of CDC6 in HBECs resulted in the re-localization of UBF (upstream binding factor) signifying rDNA [97], from nucleolar interior to the periphery, where it is associated with 53BP1 foci, forming nucleolar caps (Fig. 5c) [96].

It has been suggested that $\mathrm{R}$ loops may demonstrate dissimilar structural features depending on their role within the cell. They could be characterized as "Janus-face" modules with beneficial or adverse functions; the nature of which has not been linked with particular structural traits, yet [98]. Within this context, vimentin expression (Fig. 2c), a cardinal trait of EMT, in the "escaped" cells could be R loop-dependent, as previously reported [99]. Transcriptome analysis of the "escaped" cells revealed elevated expression of factors that prevent or remove R loops, whereas most of them were reduced during the initial phases of CDC6 induction (Fig. 5d, Additional file 10: 

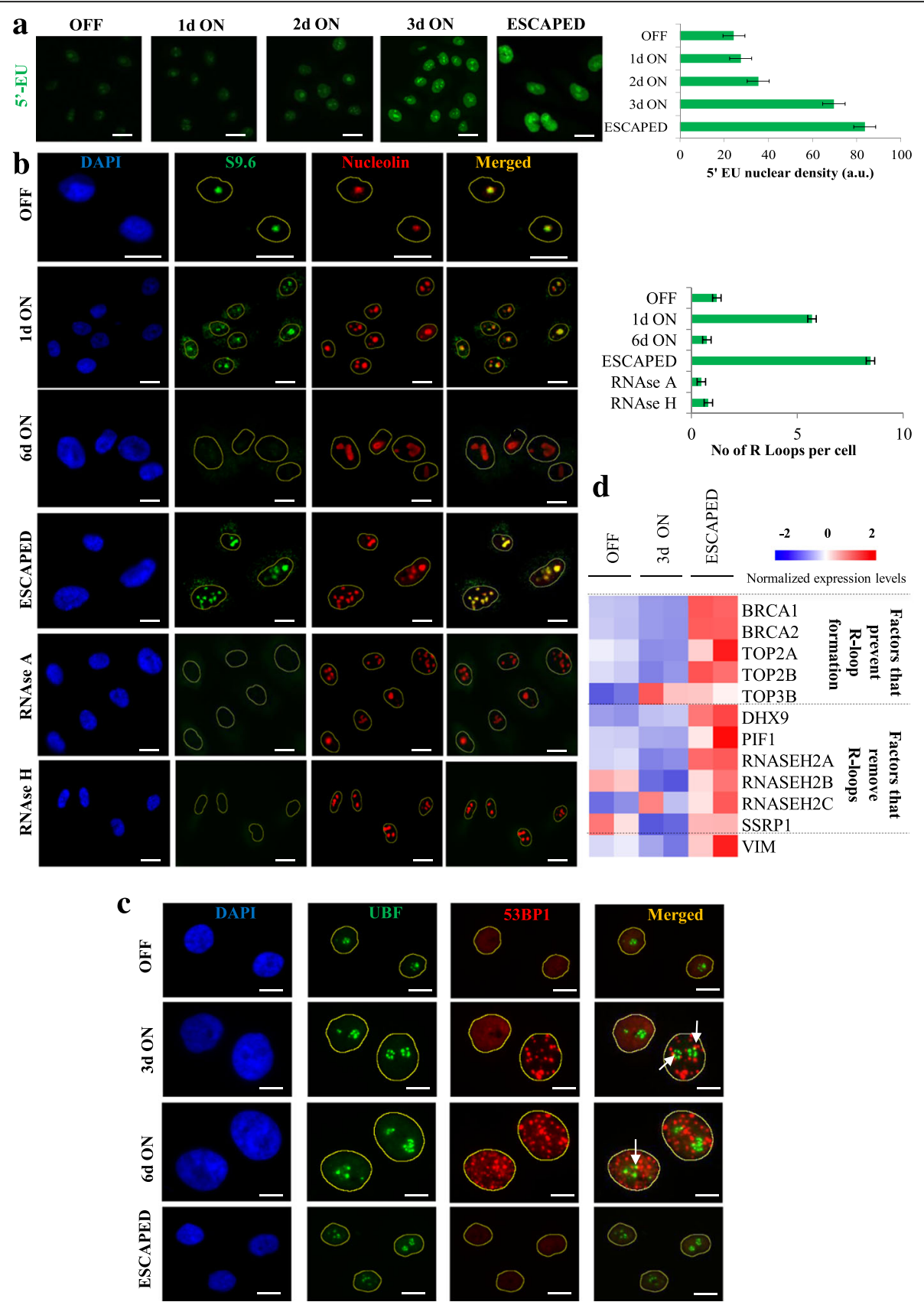

Fig. 5 CDC6 induction resulted in R loop formation. a CDC6 induction increased total transcription levels, as measured by $5^{\prime}$-EU incorporation. Scale bar: 35 mm. b IF for S9.6, antibody specific for DNA:RNA hybrids, indicated increased possibility of R loop formation upon CDC6 expression. Concurrent IF detection of nucleolin, revealed nucleolus subcellular localization of the R loops. DNA:RNA hybrids disappeared after treatment with RNase A or RNase $\mathrm{H}$ showing the specificity of the reaction. Scale bar: $15 \mu \mathrm{m}$. c Double IF for 53BP1 and UBF showed re-localization of UBF from the nucleolar interior to the periphery associated with the perinucleolar distribution of 53BP1 foci (indicated by white arrows) reflecting DNA damage repair in heterochromatin-related structures [96]. Scale bar: $10 \mu \mathrm{m}$. d Heatmap of factors affecting R loops (Additional file 11: Table S4) [98]

Figure S7 and Additional file 11: Table S4). The differential expression of these $\mathrm{R}$ loop-affecting factors in the "escaped" cells compared to early stage CDC6-induced ones may be viewed as an adaptation to the high demand for protein-synthesis of the "escaped"-protumorigenic cells; thus reducing the risk for replication-transcription collisions at the $r D N A$ loci possibly favoring the beneficial effects of the R loops.

In an attempt to reveal functional modules related to cancer initiation and progression we adjusted the data from the transcriptome and the cytogenetic analyses (Figs. 4 and 6) to the "hallmarks" of cancer $[4,73,100]$. 


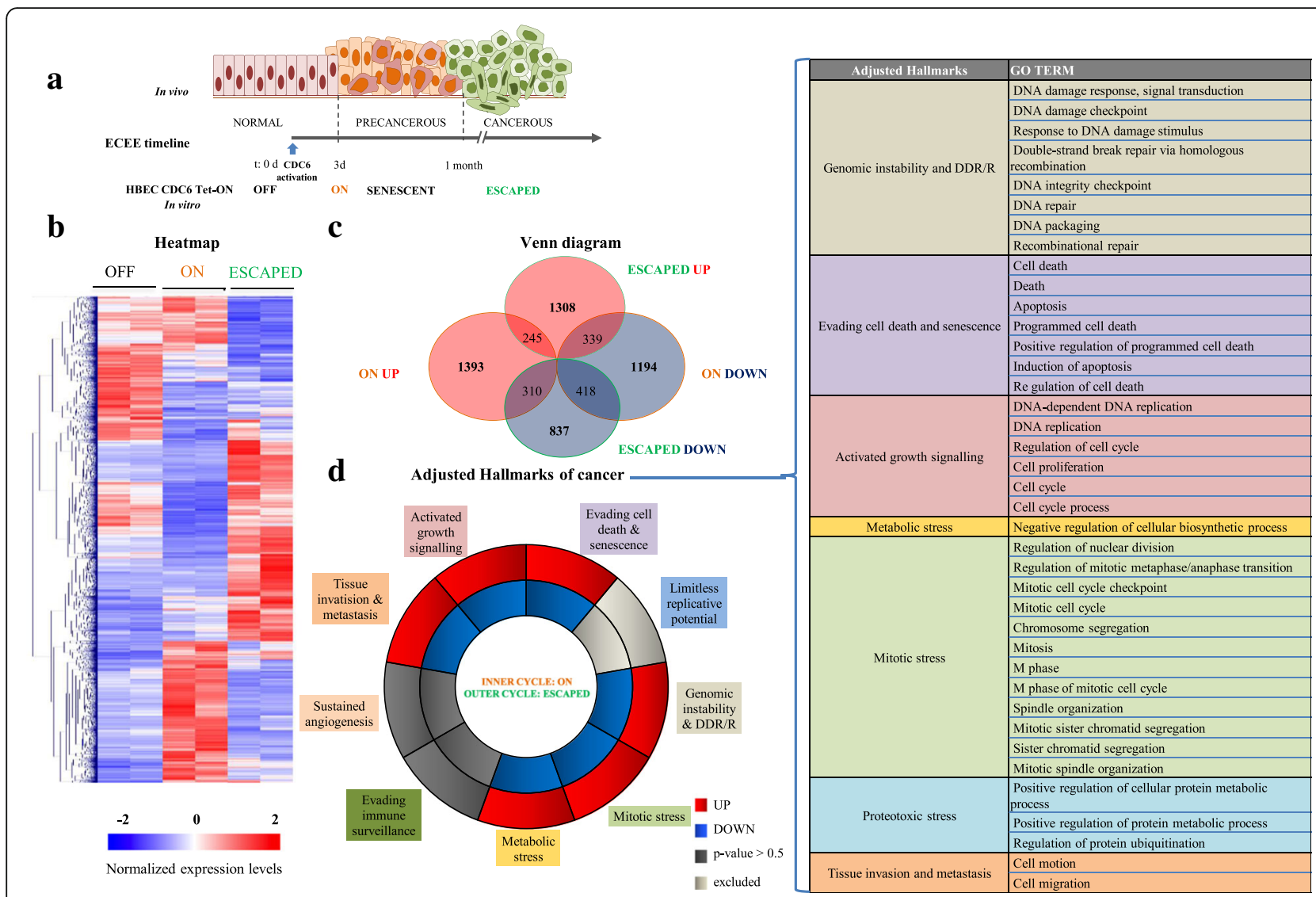

Fig. 6 Transcriptome analysis of HBEC CDC6 Tet-ON cellular system. a Timeline of ECEE (epithelial cancer evolution experiment) showing time points where main biochemical and phenotypical events occur. High-throughput RNA sequencing analysis that was performed on 3-day induced (initiation of senescence phase) and in the "escaped" cells, compared to non-induced ones, revealed extensive alterations in the transciptome landscape. $\mathbf{b}$ Heatmap, showing hierarchical clustering, and (c) Venn diagram of the deregulated genes indicated that most of them were exclusive features of either of the two time points and not common ones nominating that they share different traits. $\mathbf{d}$ Adjustment of the transcriptome analysis to the "hallmarks" of cancer, utilizing Gene Ontology (GO) terms as shown in table, revealed that the "escaped" cells share the characteristic features of cancer cells. DDR/R refers to DNA damage response and repair pathways

As depicted in Fig. 6 the results further strengthened the value of HBEC CDC6 Tet-ON cellular system to study "escape from senescence" and carcinogenesis, as the "escaped" cells share most of the cancerous characteristics, adopting a stress phenotype [101]. Of note, transcriptomics showed different transcriptome landscapes affecting major biological processes in the senescent versus the "escaped" cells (Fig. 6d). Moreover, gene-set enrichment analysis demonstrated that the cell cycle-associated pathway and DNA replication were essentially stopped in the induced cells and significantly up-regulated once the cells escaped from senescence (Additional file 12: Figure S8). In the analysis, we did not include "limitless replicative potential", as ectopic expression of hTERT could lead to artificially positive results. Notably, the only "hallmarks" that could not be scored as statistically significant were those of "evading immune surveillance" and "sustained angiogenesis", probably due to the lack of surrounding stroma.
Global miR expression analysis (miRseq) (Additional file 13: Table S5) yielded similar to RNAseq results, showing significantly different profiles between the senescent and "escaped" populations (Fig. 7). Two miRs were of particular interest: miR34c and miR29a. The first one showed significant up-regulation in the CDC6 over-expressing cells at day 3 , followed by a further rise at day 6 , whereas in the "escaped" cells, its levels fell below those observed in the non-induced cells, verified by qRT-PCR analysis (Additional file 14: Figure S9). According to reports, miR34c along with a panel of additional miRs up-regulated at day 6, are related to senescence (Additional file 15: Table S6). On the other hand, miR29a showed an inverse pattern. Of note, miR29a has been shown to play a role in EMT [102]. Interestingly, emerging data support that down-regulation of miR34 genes, including miR34c, are also implicated in EMT through a negative feedback loop with SNAIL, which comes in line with our findings [103]. Over-represented pathways, gene 


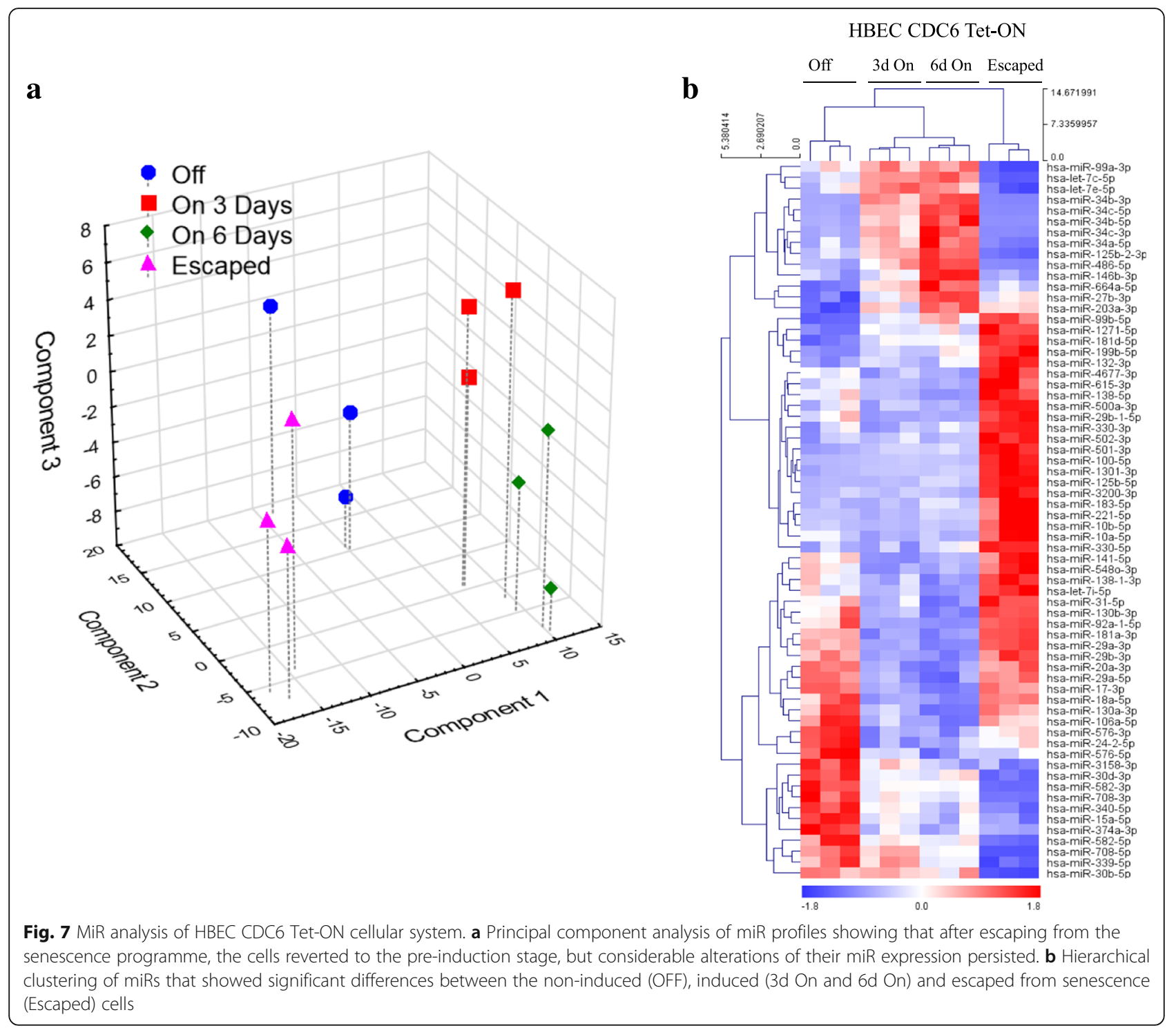

ontologies and target genes are summarized in Additional file 16: Table S7.

\section{In situ co-detection of gene coding (protein) and non-coding $(\mathrm{miR})$ products during OIS}

As mentioned, the role of miRs in senescence is well established [19, 20, 22]. However, the technical limitations of the SA- $\beta-$ Gal method (see Background section), has rendered $\mathrm{miR}$ in situ detection in senescent cells unfeasible so far. The advantages of the GL13 reagent and the described features of the HBEC CDC6 Tet-ON system (induction of senescence, escape from senescence and activation of the DDR-p53 pathway) prompted us to explore this model for monitoring, in situ, the spatiotemporal expression pattern of a gene coding (protein) and non-coding (miR) product functionally linked with the processes of DDR and senescence. Prompted by the
miRseq analysis we selected miR34c as a miR target. MiR34a-c are amongst the best-characterized direct transcriptional downstream targets of p53, placing them as bona fide components of the p53 network [104]. In addition, miR-34s, including miR34c, were reported to trigger senescence in various human lung settings [104, 105]. On the other hand, 53BP1 is an ideal protein target since it is a well-established upstream mediator of the DDR pathway, which is recruited to sites of double strand breaks (DSBs) forming discrete foci [55, 106-108]. For in situ co-detection we followed a three-step immunofluorescence process: i) Fluorescence in situ hybridization (FISH) for miR34c, followed by ii) GL13 staining to spot senescent cells, and finally iii) detection of 53BP1 foci. Successful GL13 staining and 53BP1 foci formation (steps ii and iii) were independently confirmed during the validation of the HBEC CDC6 Tet-ON 


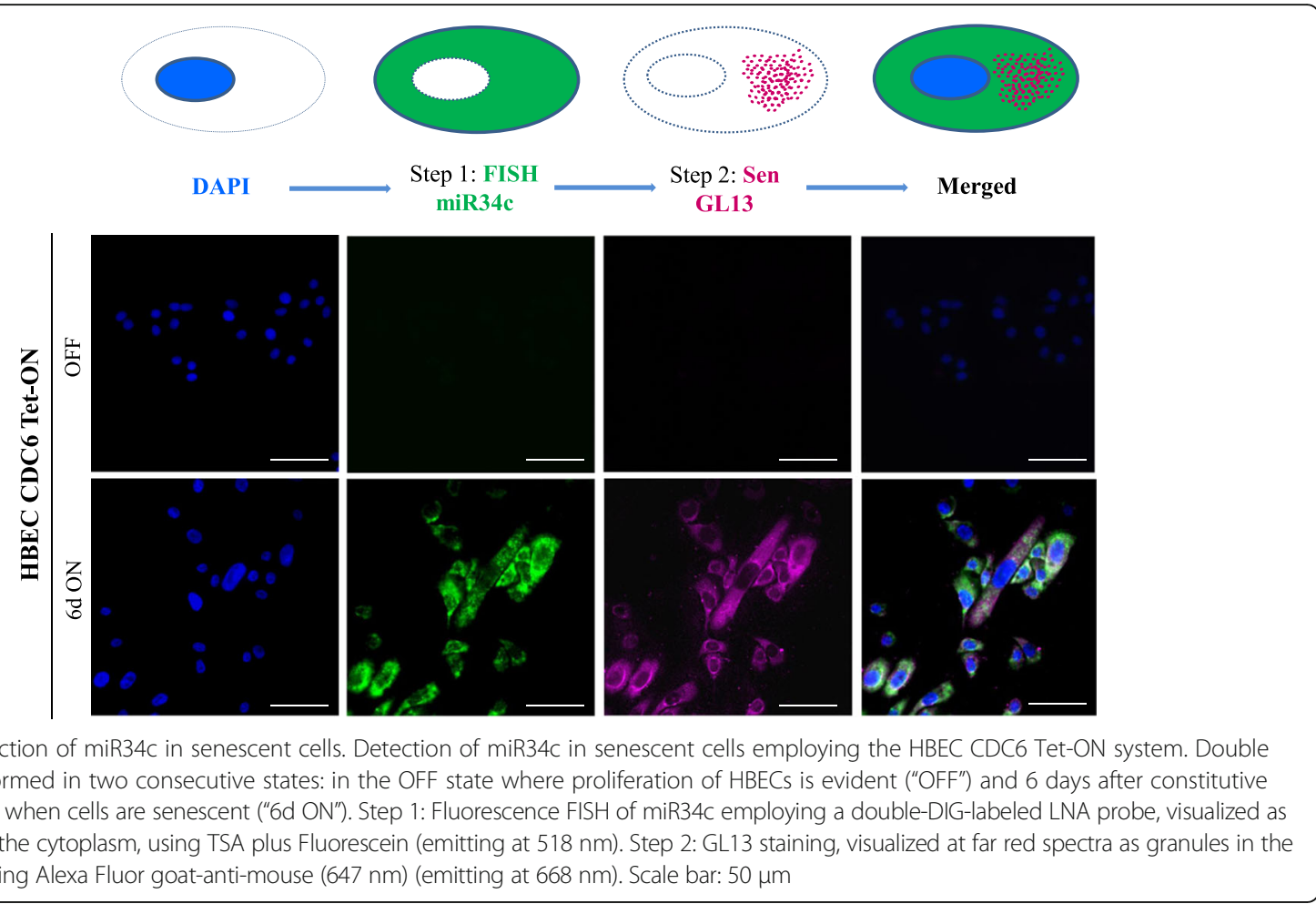

system, as shown in Figs. 1d and 3c and Additional file 7: Figure S4b, respectively.

Due to the challenging nature of the 3-step codetection procedure, detailed technical aspects, control and trouble-shooting processes are described meticulously in the Additional file 17 and illustrated in Additional file 18: Figure S10. Only the principles of the in situ assay are presented and discussed in the current section. Step 1; miR34c detection: Before proceeding with ISH of miR34c, its expression following CDC6 induction was confirmed by qRT-PCR (Additional file 14: Figure S9). For ISH we employed the Locked Nucleic Acid (LNA) technology [109-111]. LNAs are nucleic acid analogues "locked" by a methylene bridge that constrains them in the ideal conformation for Watson-Crick binding allowing superior hybridization properties [112] (Additional file 19: Figure S11). To increase detection sensitivity we used double-digoxigenin (DIG) labeled (at both $5^{\prime}$ and $3^{\prime}$ ends) probes visualized with the Tyramide Signal Amplification (TSA) Plus Fluorescein System emitting at $517 \mathrm{~nm}$ [113-115]. Although the water-soluble 1-ethyl-3-(3-dimethylaminopropyl) carbodiimide (EDC) increases signal strength, we omitted it since it interferes with protein detection [116]. As negative and positive controls of the method we employed Double-DIG labeled Scramble-miR and U6 small nuclear RNA (snRNA) probes, respectively (Additional file 20: Figure S12). Step 2; Detection of senescent cells: To detect miR34c positive senescent cells we employed the GL13 reagent [53] that overcomes the restrictions of the SA- $\beta-$ Gal assay (see Background section) allowing examination of the sensitive to decay miR molecules to precede senescence detection. Step 3; Detection of 53BP1 foci: Finally, we proceeded with 53BP1 foci detection using conventional immunofluorescent (IF) analysis. Each secondary antibody emitted at different wave-lengths to avoid overlapping during visualization (see Methods section, Figs. 8 and 9).

As depicted in Figs. 8 and 9 following a two (steps 1 and 2) and a three step (steps 1,2 and 3) process we successfully co-detected miR34c in senescent cells that clearly showed evidence of DDR activation (53BP1 foci). For each step a parallel experiment took place, omitting the primary reagent (miR34c probe, GL13 and anti53BP1 antibody), to exclude false positive staining from the secondary antibodies. Notably, miR34c was not detected in the "escaped" cells (Fig. 9), which is in agreement with the miRseq and qRT-PCR analysis (Additional file 14: Figure S9), probably because of declined p53 levels in these cells (Fig. 3c).

\section{Discussion}

A major challenge in understanding the pathogenesis of cancer is the availability of models that functionally recapitulate observations from human clinical settings. A successful model addresses the following questions: How relevant is it to human cancer? What type of human cancer does it mimic? Is the recapitulated event an initiating or a promoting one? How can it help 




uncover or predict mechanism(s) that could be therapeutically targeted?

While in vivo mice models are the first choice, their development is costly and time consuming. Moreover, their relevance to human carcinogenesis has been questioned despite the large body of knowledge gained till now [117]. In mice the quantity of genetic events required for cellular transformation is lower compared to humans $[100,118]$, whereas qualitative alterations differ, as well. As an example, in humans activated oncogenes are encountered mainly by the DDR pathway, while in mice activation of the p19ARF tumorsuppressor predominates [58, 59, 119-121]. Additionally, telomeres in humans are shorter than in mice, possibly 
as an anti-tumor protective mechanism to eliminate cells that have acquired dangerous mutations during the relative long human lifespan [122].

Human cell lines represent established alternatives as they are more relevant to human disease, although there are certain limitations, such as the absence of complex intercellular and cell-matrix interactions that exist in in vivo models [100]. Still, they facilitate the direct interrogation of cancer associated genetic events, like oncogene activation, and their impact on cellular outcomes, such as evasion from the anti-tumor barriers of apoptosis and senescence [36].

In this study, we developed and present an original human non-malignant epithelial (bronchial) oncogeneinducible cellular system that recapitulates the precancerous and cancerous phases of epithelial carcinogenesis precisely, within a relatively short time frame (Figs. 1 and 6a). Non-malignant epithelial platforms are generally rare as most researchers prefer fibroblast- or cancer cell linebased settings because they are manipulated much easier. However, the cellular context determines to a great extent the carcinogenetic process. Given that the majority of cancers are of epithelial origin, non-malignant epithelial systems are essential to study the initiating events of epithelial carcinogenesis. Prompted by the fact that our model showed a potent senescent response, following oncogene activation, we took advantage of GL13, a novel senescence biomarker we synthesized, and monitored concurrently in situ miR and protein expression, for the first time, during OIS. The latter was not feasible until now due to inherited flaws of SA- $\beta-$ Gal, the currently available method [40], whereas GL13 enables detection of senescent cells in any given biological material [53].

As a triggering oncogenic stimulus we favored the replication licensing factor CDC6 mainly because it is frequently over-expressed in various epithelial cancers from their earliest stages of development [60,61]. Moreover, we previously showed that its deranged expression is not a mere reflection of increased proliferation but a potential "driving force", as suggested by the oncogeneinduced replication stress model for cancer development, we proposed few years ago [33, 36, 62]. Lying in the heart of the replication machinery we anticipated that CDC6 induction in the HBEC setting would mimic from an evolutionary perspective the precancerous and cancerous stages of epithelial cancer development.

Indeed, CDC6 over-expression provoked a rapid senescent response that was maintained for a prolonged period (precancerous phase) (Fig. 1 and Additional file 7: Figure S4). In parallel, at the molecular level an intense DDR response was noticed, which is in line with previous reports demonstrating a functional link between DDR and acquirement of senescence [33, 34, 64, 72, 74]. The most interesting finding appeared at day 30 post- induction when cellular clones with phenotypical and molecular traits of EMT emerged ("escaped" cells cancerous phase) (Figs. 1d and 2c and Additional file 7: Figure S4). EMT is an embryonic program that confers to cancer invasion and progression when reactivated at the "wrong time" [70, 71]. The DDR was alleviated in the "escaped" cells implying that an extensive repair process occurred. Judging from the novel clonal and random chromosomal alterations observed in the "escaped" cells (Fig. 4 and Additional file 8: Figure S5), an errorprone repair procedure probably took place, the exact nature of which remains to be elucidated. A similar process has been recently described during chronic p21 ${ }^{\text {WAF1/Cip } 1}$ expression in a p53-independent environment [64]. In addition to the EMT features, the "escaped" cells possessed most of the hallmarks of cancer, as depicted from the transcriptome analysis of the "escaped" versus the non-induced cells (Fig. 6d), clearly pinpointing the value of our cellular system to study induction and escape from senescence.

Apparently and in agreement with the oncogeneinduced replication stress model for cancer development, CDC6-driven genomic instability exerted a selective pressure that led to the evolution of the "escaped" cells. The fact that this process was raised during the senescent phase puts forward a scenario according to which senescence should not be viewed as a "static" state, but as a "dynamic" one, when the genome landscape is continuously reformed and shaped. Although re-replication is "incriminated" as the means by which CDC6 causes DNA damage, the current study unraveled that $\mathrm{R}$ loops could represent and additional source of CDC6 mediated genomic instability [83]. R loop formation occurred within the nucleoli, heterochromatin nuclear substructures comprised by the $r D N A$ region. The $r D N A$ locus is a highly repetitive region of the genome consisting of tandem repeats encoding the rRNA subunits and is enriched with origins of replication. The latter along with the fact that CDC6 was shown to activate the transcription of $r D N A$ [66] renders its loci prone to $\mathrm{R}$ loop formation, replication-transcription collision and DNA damage. In support to this notion, we observed in CDC6-induced HBECs redistribution of UBF to the periphery of the nucleoli localized adjacent to 53BP1 foci, forming the so-called nucleolar caps (Fig. 5c) [96]. Having in mind that UBF is the main transcription factor of $r D N A$ [123] and repair of heterochromatin DNA takes place at its periphery [95, 124], we can deduce that R-loop formation in our setting most probably led to DNA damage; thus contributing to CDC6-driven genomic instability.

Conclusively, we believe that the experimental system we present can inspire further mechanistic studies and address questions such as which origins of replication deregulated CDC6 activates. Are they intergenic or intrangenic? In the latter case what is the risk of replication-transcription 
collision? What type of error-prone repair pathway(s) ensues during CDC6 induced senescence? Are the transcriptional properties of deranged $\mathrm{CDC6}$ restricted only to the INK4/ARF, CDH1 (E-cadherin) and $r D N A$ loci or does CDC6 mediate a global transcriptional program? If yes, does it involve displacement of the chromosomal insulator CTCF as it does for the INK4/ARF and CDH1 loci? Moreover, with regard to the miRseq analysis, which of the detected miRs are directly involved in inducing senescence and which are implicated in the escape phenomenon? All the above questions and their answers gain particular worth within the context of the non-malignant epithelial environment of our system as they most probably signify cancer initiating events.

\section{Conclusions}

The desire to simultaneously detect multiple intracellular macromolecules has its roots back to late 60's [125]. Despite progress in the development of in situ detection assays during the last decades, multiple in situ staining still remains a challenge. This is pertinent to the field of senescence because of the additional practical limitations exerted by the application of the SA- $\beta-G a l$ assay. Prompted by this widespread need, we have developed and described herein a methodology that bypasses these restrictions. We have provided proof-of-principle that this methodology maintains optimal preservation of the biological setting and tremendous flexibility, enabling gold standard ISH techniques to be combined with antigen detection and senescence marker recognition.

To validate the new method, we have developed a cell system based on human bronchial epithelial cells that recapitulates the whole spectrum of epithelial carcinogenesis following the inducible over-expression of the replication licensing factor CDC6. The vast majority of in vitro models used to study processes related to malignant transformation are fibroblast-based, which contrast the fact that most malignancies are of epithelial origin. Thus, the HBEC CDC6 Tet-ON epithelial system emerges as a valuable prototypical tool that possesses the following advantages: i) It enables phenotypic and molecular monitoring of OIS and escape from senescence, recapitulating the precancerous and cancerous phases of human epithelial carcinogenesis. In the future it could be applied in co-cultures with stromal cells; hence investigating the interplay between the various phases of OIS and the surrounding cellular environment. ii) The properties of CDC6 acting concurrently as a replication and transcription factor, when deregulated, will facilitate the in-depth study of genome dynamics, such as re-replication, replication-transcription collision, DNA repair in conjunction with transcriptional deregulation, and chromatin remodeling. Along these lines we have observed temporal formation of $\mathrm{R}$ loops that cease during senescence and reappear upon escape. iii) Applying the senescence detecting reagent GL13, which lacks the limitations of the SA- $\beta-G a l$ assay, provides the unique opportunity to monitor co-currently in situ both proteins and regulatory RNAs, such as miRs. Moreover, the utilization of the GL13 compound for detection of lipofuscin is applicable not only to track senescence, but also to monitor escape from it. The latter is due to the fact that lipofuscin is non-degradable, accumulating progressively and is diluted only through cell divisions. As a result, traces of lipofuscin in the cells escaping from senescence can be detected rendering it a unique lineage tracing marker [126]. We expect that this cell model, coupled with the multifaceted applications that the new macromolecule detection methodology offers, will enable a more direct examination of the spatiotemporal expression of miRs and gene-coding products relevant to senescence and malignant transformation.

\section{Methods \\ Cell culture, plasmids and HBEC CDC6 Tet-ON system generation}

The Lenti- $\mathrm{X}^{\mathrm{ma}}$ Tet-On ${ }^{\circ}$ 3G Inducible Expression System (Clontech Laboratories) was employed to establish a CDC6 inducible-expression cellular model in immortalized HBECs (hTERT/CDK4) [57], which were a kind gift of Dr. T. Liloglou.

PLVX-TRE3G-CDC6 was generated by digesting pTRE2Hyg-CDC6 [63] with BamHI and EcoRV (blunt end) and subcloning MYC-tag-hCDC6-cDNA into PLVXTRE3G linearized with BamHI and SmaI (blunt ended). Immortalized HBECs are resistant to G418 due to the neomycin-resistant gene introduced with the CDK4 expression vector (pSR $\alpha-\mathrm{MSU}$ ) and to puromycin due to the corresponding-resistant gene introduced with $\mathrm{p}$-babehygro-hTERT. Given that PLVX-TET3G and PLVX3GTRE-CDC6 carry also neomycin-resistant and puromycinresistant genes, accordingly, it was necessary to replace the resistance cassettes of lentiviral vectors with blasticidin-resistant (BSD) and zeocin-resistant (BleoR) genes. Particularly, IRES-BSD from pBIB was transferred into pBluescript SK with EcoRI-ClaI and then obtained with KpnI and BamHI to replace IRES-Neo-WPRE from PLVX-TET3G using partial digestion. BleoR was derived from Lenti X1 zeo-pTERshATM with XbaI-filled ends and KpnI to replace puromycin-restistant cassette of PLVX3G-TRE-CDC6 obtained with MluI-filled ends and KpnI. For a schematic presentation of vectors see Fig. 1a.

Production of lentiviruses and transduction were performed according to supplier's guidelines utilizing Lenti- $\mathrm{X}^{\mathrm{nt}}$ Concetrator (Clontech Laboratories). After two-week selection with $3 \mu \mathrm{g} / \mathrm{mL}$ blasticidin and $12.5 \mu \mathrm{g} / \mathrm{mL}$ zeocin, cell clones with doxycyclin-dependent $(1 \mu \mathrm{g} / \mathrm{mL}$, Sigma $)$ induction of CDC6-MYC-tagged, as assessed by 
immunoblot and real-time qRT-PCR analyses (Fig. 1a), were isolated and used for the described experiments. Upon CDC6 induction, doxycyclin was replenished every second day.

Immortalized HBECs and HBEC CDC6 Tet-ON cells were maintained in Keratinocyte Serum-Free Medium (\#17005-075, Invitrogen) supplemented with $50 \mu \mathrm{g} / \mathrm{ml}$ Bovine Pituitary Extract and $5 \mathrm{ng} / \mathrm{ml}$ hEGF (\#17005-075, Invitrogen) at $37^{\circ} \mathrm{C}$ and $5 \% \mathrm{CO}_{2}$ [57].

Microphotographs were obtained with an inverted microscope (Axiovert S100; Carl Zeiss) equipped with $\mathrm{CP}$-Achromat objectives and a charge-coupled device IRIS colour video camera (SSC-C370P; Sony), using Image Pro Plus v3.0 (Media Cybernetics) software.

\section{Total protein extraction and western blot analysis}

Total protein extracts were obtained by homogenization in $50 \mathrm{mM}$ Tris/ $\mathrm{HCl} \mathrm{pH} \mathrm{8.0,} 150 \mathrm{mM} \mathrm{NaCl}, 0,1 \%$ SDS, $0,5 \%$ sodium deoxycholate, $1 \% \mathrm{NP}-40$ adjusted with protease and phosphatase inhibitors. The homogenate was centrifuged at $13,400 \mathrm{rpm}$ at $4{ }^{\circ} \mathrm{C}$ for $10 \mathrm{~min}$. The supernatant was collected and protein content quantified using Protein assay dye concentrate (BIO-RAD). Thirty $\mu \mathrm{g}$ of protein from total extracts, were adjusted with Laemmli Buffer (Sigma) and loaded on acrylamide/bis-acrylamide gels. Gel electrophoresis, transfer to PVDF membrane (Millipore) and signal development with chemiluminescence substrate ECL for HRP (PerkinElmer) were performed as previously described [60]. Primary antibodies were used at the following dilutions: CDC6 (Santa Cruz \#9964) 1:1000, p-RB (Santa Cruz \#7986-R) 1:500, RB (Santa Cruz \#50) 1:1000, p53 (Santa Cruz \#47698) 1:1000, p21 WAF1/Cip1 (Santa Cruz \#6246, 1:400), Actin (Cell Signaling \#4967, 1:1000). Anti-mouse (Cell Signaling \#7076) and anti-rabbit (Cell Signaling \#7074) HRP-linked secondary antibodies diluted at 1:1000 were used.

\section{RNA isolation, CDNA preparation and qRT-PCR}

RNA was extracted using Nucleospin RNA (MachereyNagel \#740955) according to the manufacturer's instructions. $1 \mu \mathrm{g}$ RNA was used for cDNA preparation with Primescript $^{\text {tm }}$ RT Reagent Kit (Takara \#RR037A). Real-time qRT-PCR analysis was performed utilizing SYBR Select Master Mix (Life technologies \#4472908) on a DNAEngine-Opticon (MJ-Research) thermal cycler. Primer sequences are as follows, CDC6 forward: 5'-CAGTT CAATTCTGTGCCCGC-3' and reverse: 5'-GCTCCTTCT TGGCTCAAGGT-3', $\beta 2$-microglobulin (reference gene) primers were forward: 5'-TCGCGCTACTCTCTCTTTCT-3' and reverse: 5'-TTTCCATTCTCTGCTGGATGAC-3'. Results, averaged from three independent experiments, are presented as $\mathrm{n}$-fold changes for the various time points after CDC6 induction versus the values of the noninduced sample, using the $2-\Delta \Delta^{\mathrm{CT}}$ method.
For miR detection, RNA was extracted with the NucleoSpin miR, kit/50preps (Cat no: 740971.50; Macherey-Nagel), following the manufacturer's instructions. For reverse transcription the TaqMan ${ }^{\circ}$ MicroRNA RT Kit, 200 RXNS (Cat no: 4366596, ThermoFischer Scientific) along with $\mathrm{TaqMan}^{\circ}$ Universal Master Mix II with UNG (Cat no: 4440042, ThermoFischer Scientific) and the two TaqMan ${ }^{\circ}$ MicroRNA Assays Inv sM10 (Cat no: 4427975, ThermoFisher Scientific) were employed. The assays ID for each of the corresponding primers and probes are the following: a) hsa-miR-34c (target assay): 000428 and b) U6snRNA (control assay): 001973. Expression of miR-34c was calculated relative to U6snRNA levels according to the comparative method of $2^{-}{ }^{\Delta \Delta C T}$. Relative quantification of $\mathrm{miR}$ expression was calculated from three independent biological replicates.

\section{ChIP assay}

ChIP assay was performed in HBEC CDC6 Tet-ON cells grown in $100 \mathrm{~mm}$ plates and induced for 2 days. Cells were cross-linked with $1 \%$ formaldehyde in $50 \mathrm{mM}$ Hepes- $\mathrm{KOH}$ pH 7.5, $100 \mathrm{mM} \mathrm{NaCl}, 1 \mathrm{mM}$ EDTA $\mathrm{pH}$ 8.0, $0.5 \mathrm{mM}$ EGTA $\mathrm{pH} 8.0$ for $15 \mathrm{~min}$ at RT on a rocking platform. Cross-linking was stopped by the addition of glycine to a final concentration of $125 \mathrm{mM}$ for $5 \mathrm{~min}$ at RT on a rocking platform. Cross-linked cells were washed twice with ice cold PBS, scraped and centrifuged at $3000 \mathrm{rpm}$ for $5 \mathrm{~min}$. Approximately $6 \times 10^{6}$ cells were resuspended in $2 \mathrm{ml}$ of lysis buffer $(50 \mathrm{mM}$ Hepes pH 7.9, $140 \mathrm{mM} \mathrm{NaCl}, 1 \mathrm{mM}$ EDTA, 10\% glycerol, 0.5\% NP-40, 0.25\% Triton-X100) and incubated for $10 \mathrm{~min}$ on ice in the presence of protease and phosphatase inhibitors. Cells were then washed twice in $10 \mathrm{mM}$ Tris- $\mathrm{HCl} \mathrm{pH}$ 8.1, $200 \mathrm{mM} \mathrm{NaCl}, 1 \mathrm{mM}$ EDTA $\mathrm{pH}$ 8.0, $0.5 \mathrm{mM}$ EGTA pH 8.0, two more times in shearing buffer $(0,1 \%$ SDS, $10 \mathrm{mM}$ Tris- $\mathrm{HCl} \mathrm{pH} 8.1,1 \mathrm{mM}$ EDTA pH 8.0) and resuspended in $1 \mathrm{ml}$ of shearing buffer in the presence of protease and phosphatase inhibitors. Cells were sonicated with a Covaris S2 sonicator for 15 min. Triton-X100 and $\mathrm{NaCl}$ was added to a final concentration of $1 \%$ and $150 \mathrm{mM}$, respectively. The debris was pelleted by 10 -min centrifugation at $13000 \mathrm{rpm}$ at $4{ }^{\circ} \mathrm{C}$, and the soluble chromatin material was precleared with salmon sperm DNA/50\% protein A agarose slurry. Further steps were performed as described before [63].

\section{BrdU proliferation assay}

For BrdU incorporation, cells grown on coverslips were pulse-labeled with $10 \mu \mathrm{M}$ BrdU (Roche) overnight at $37{ }^{\circ} \mathrm{C}$. Cells with incorporated BrdU were treated and visualized by indirect IF analysis as described in the corresponding methodology subsection. 


\section{Senescence/GL13 staining}

GL13 staining was performed as described before [53]. GL13 compound is commercially available as SenTraGor $^{\text {TM }}$ from Arriani Pharmaceuticals (Cat no: AR8850040).

\section{SA- $\beta$-gal assay}

SA- $\beta-$ Gal activity was detected according to DebacqChainiaux et al. [127].

\section{Wound healing assay}

Cells were seeded on $100 \mathrm{~mm}$ tissue-culture plastic dishes at $70 \%$ confluence and, the next day, a scratch wound was performed using a sterile $200 \mu \mathrm{l}$ pipette tip. Phase-contrast images were taken at the starting $(0 \mathrm{~h})$ time point and at $8 \mathrm{~h}$ and $24 \mathrm{~h}$ time intervals using an inverted microscope (Axiovert S100; Carl Zeiss).

\section{Electron microscopy analysis}

Cells were fixed in a freshly-prepared solution containing 3\% formaldehyde (prepared from paraformaldehyde) and $0.5 \%$ glutaraldehyde in $0.1 \mathrm{M}$ phosphate buffer, $\mathrm{pH} 7.4$, for $30 \mathrm{~min}$ at room temperature (RT). Cells were then harvested using a scraper, collected into a tube and centrifuged at $800 \mathrm{~g}$ for $5 \mathrm{~min}$ at RT. The supernatant was aspirated, while cells were resuspended in $4 \%$ gelatin warmed aquatic solution followed by a spin down at $800 \mathrm{~g}$ for $5 \mathrm{~min}$ at RT and cooled on ice. Under a stereoscope the solidified cell pellet with gelatin was extracted, cut into small fragments $\left(1-2 \mathrm{~mm}^{3}\right)$ and transferred into $0.1 \mathrm{M}$ phosphate buffer, $\mathrm{pH} 7.4$ at $4{ }^{\circ} \mathrm{C}$. The cell-gelatin fragments were then dehydrated in graded series of ethyl alcohol, followed by propylene oxide (PO) treatment, infiltrated gradually in a mixture of Epon/Araldite resins diluted in PO and finally embedded in fresh epoxy resin mixture. Ultrathin epoxy sections (70-90 nm thickness) were cut on a Leica Ultracut $\mathrm{R}$ ultramicrotome, equipped with a Diatome diamond knife, and mounted onto 200-mesh copper grids. Ultrathin sections were observed with a Philips 420 transmission electron microscope and micrographs were taken with an Olympus Megaview G2 CCD camera.

\section{Indirect IF analysis}

For indirect IF analysis cells were grown on coverslips and fixed with $100 \%$ ice-cold methanol or $4 \%$ formaldehyde (prepared from paraformaldehyde) for $10 \mathrm{~min}$ and store at $4{ }^{\circ} \mathrm{C}$ until staining was performed. Following, cells were permeabilized with $0,3 \%$ Triton X-100 in PBS for 5 min at RT. A $10 \%$ fetal bovine serum and 3\% bovine serum albumin in PBS solution was used as a blocking buffer for $1 \mathrm{~h}$ at RT. Primary antibodies were diluted in blocking buffer and incubated overnight at $4{ }^{\circ} \mathrm{C}$. Secondary antibodies were goat anti-rabbit or goat anti-mouse, Alexa Fluor ${ }^{\circledR} 488$ or Alexa Fluor 568 (Invitrogen) diluted 1:500 in blocking buffer. Counterstaining was performed with $100 \mathrm{ng} / \mathrm{ml}$ DAPI (Sigma-Aldrich). Primary antibodies used were: CDC6 (Santa Cruz \#9964, 1:100 or \#8341, 1:50), Cyclin A (Santa Cruz \#239, 1:150), E-cadherin (Cell Signaling \#3195 1:200), Vimentin (Dako M0720, 1:50) 53BP1 (abcam \#21083, 1:250), S9.6 (Kerafast ENH001, 1:200), nucleolin (Cell Signaling \#14574, 1:1000) and UBF (Santa Cruz \#13125, 1:100). For S9.6 IF, treatment with RNase A $(0.25 \mu \mathrm{g} / \mu \mathrm{l})$ or RNase $\mathrm{H}$ (50 units/slide) at $37{ }^{\circ} \mathrm{C}$ for $30 \mathrm{~min}$ and $3 \mathrm{~h}$, respectively, was performed if necessary. Image acquisition from multiple random fields was automatically obtained on a ScanR screening station (Olympus, Germany) and analyzed with ScanR (Olympus, Germany) software, or a Zeiss Axiolab fluorescence microscope equipped with a Zeiss Axiocam MRm camera and Achroplan objectives, while image acquisition was performed with AxioVision software 4.7.1.

\section{Flow cytometric analysis (FACS)}

Cell cycle analysis was assessed on a FACS Calibur (Becton-Dickinson) as previously described [63].

\section{Comet assay}

Comet assay was performed with minor modifications of a standard previously described protocol [63]. In brief, after trypsinization and wash in PBS, 200,000 viable cells were resuspended in $225 \mu$ of TBE (Tris-Boric acid-EDTA) buffer, mixed with $1275 \mu$ l of low melting agarose embedded in plugs. Plugs were subsequently incubated in $10 \mathrm{ml}$ lysis solution (100 mM Tris- $\mathrm{HCl}, 100 \mathrm{mM}$ EDTA, and $2.5 \mathrm{M}$ $\mathrm{NaCl}, \mathrm{pH} 10$, with the addition of $1.25 \%$ Triton $\mathrm{X}-100$ and $10 \%$ DMSO, before use) overnight on ice and in the dark. After completion of lysis, plugs were washed twice in TBE for $30 \mathrm{~min}$ on ice in the dark. Finally, plugs were washed and subsequently incubated in ice-cold alkaline denaturation buffer (300 mM NaOH and 1 mM EDTA, pH 13) for $45 \mathrm{~min}$ on ice in the dark. For electrophoresis, plugs were mounted onto $1 \%$ agarose-coated slides that were placed into a $30-\mathrm{cm}$ horizontal constant-field gel electrophoresis chamber in ice-cold alkaline denaturation buffer for $30 \mathrm{~min}$ at $0.7 \mathrm{~V} / \mathrm{cm}$ and at $4{ }^{\circ} \mathrm{C}$. After electrophoresis, slides were washed 3 times in TBE, dehydrated in ice-cold ethanol (100\%) for $15 \mathrm{~min}$ and then allowed to dry in the dark. $24 \mathrm{~h}$ later, slides were rehydrated in $5 \mathrm{ml}$ of deionized water for $10 \mathrm{~min}$, and $40 \mu \mathrm{l}$ of diluted SYBR gold (Invitrogen, Molecular Probes) was applied on each plug. Cells were observed under a fluorescence microscope (Axiolab) equipped with a monochrome UV camera (XC-EU50 CE; Sony). Analysis was conducted using the CometScore software (TriTek Corp.).

\section{5'-EU incorporation based nascent RNA assay}

In situ detection of nascent RNA was performed with the Click-iT Alexa Fluor 488 Imaging Kit (Invitrogen, 
Molecular Probes). Briefly, cells were incubated for $30 \mathrm{~min}$ in the presence of $0.5 \mathrm{mM} 5$-EU. Samples were fixed in $4 \%$ formaldehyde for $15 \mathrm{~min}$ and permeabilized in $0.5 \%$ Triton X-100 for $20 \mathrm{~min}$ at RT. Samples were then processed according to the manufacturer's recommendation. Cells were analyzed using LSM780 or LSM710 (Carl Zeiss Microscopy) confocal microscopes and 5-EU nuclear intensity was quantified with the NISelements software (Nikon).

\section{Molecular Cytogenetics}

Molecular cytogenetics analysis was conducted as previously published [63]. Specifically, a $63 \times$ magnification lens on a fluorescent Axio-Imager Z1, Zeiss microscope equipped with a MetaSystems charge-coupled device camera and the MetaSystems Isis software were used.

\section{RNA-sequencing (RNAseq) and miR-sequencing (miRseq) preparation and analysis}

The library preparation for RNAseq and miRseq was carried out in the Greek Genome Center (GGC) of Biomedical Research Foundation of Academy of Athens (BRFAA). RNA was collected from biological duplicates of HBEC CDC6 Tet-ON non-induced (OFF), 3d ON and "escaped" cells. RNAseq libraries were prepared with the TruSeq RNA kit using $1 \mu \mathrm{g}$ of total RNA. The libraries were constructed according to Illumina's protocols and then were mixed in equal amounts. Paired-end 38 bp reads for 2 "OFF", 2 "ON" and 2 "ESCAPED" status samples were generated with NextSeq500 in the GGC. RNAseq raw sequencing data were aligned to human genome version GCCh37/hg19 with the use of tophat (version 2.0.9) [128] and the use of «-b2-verysensitive» parameter. Data filtering and file format conversion was performed with Samtools (version 0.1.19) [129]. Aligned reads were assigned into exons using HTseq count (version 0.6.1p1) algorithm [130] with the following command «htseq-count $-\mathrm{s}$ no $-\mathrm{m}$ intersection -nonempty». Finally differentially expressed genes were identified with the use of DESeq $R$ package [131] and genes with at least 10 reads, fold change cut off 1.5 and $p$-value $\leq 0.05$ were considered to be differentially expressed (DEGs) (Additional file 11: Table S4).

For miRseq the procedure was carried out differently. After automatic filtering, adapter trimming, error removing and quality control using AfterQC [132], good quality reads were mapped directly to miRbase (release 21) database of all mature miR sequences using Novocraft's NovoAlign tool. This allowed us to create raw count tables accounting for every present miR. Raw counts were then normalized using standard transcripts per million (tpm) normalization. Length of miRs was acquired from miRbase v21. Four groups were compared: non-induced cells (OFF), induced ones (ON at 3 and
6 days) and "escaped" from senescence (ESC). MiRs present in at least 10 transcripts per million in at least three samples from any of the groups were selected for comparisons, yielding the total number of 269 unique miRs ready for analysis. The four groups were compared with one-way ANOVA. MiRs with Bonferroni-adjusted $p<0.05$ were entered into a hierarchical clustering and principal component analyses (Additional file 13: Table S5). Post-hoc comparisons between the groups were performed using a Tukey's test.

\section{Bioinformatic and functional analyses}

DAVID knowledgebase [133] was used for Gene Ontology analysis. Only pathways and biological processes with p-value $\leq 0.05$ (Fisher's exact test) were considered to be significantly enriched. RNAseq data have been deposited in the Short Read Archive (SRA) under the accession codes PRJNA388146. Gene set enrichment analysis (GSEA) [134] was performed using the canonical pathways $(\mathrm{Cp} 2)$ subset of the molecular signatures database [http://software.broadinstitute.org/ gsea/msigdb/].

For miRs, the over-representation analysis was performed using miRNA enrichment analysis miEAA [135]. Over-represented pathways and gene ontologies were searched using the miRWalk and the search for target genes utilized the miRTarBase as reference. To account for multiple comparison problems we lowered the threshold for statistical significance to 0.001 as it was impossible to fully adjust for multiple hypothesis testing and the number of between group analyses. Additionally, the threshold of at least $3 \mathrm{miRs}$ present in the analyzed sets was applied. MiRseq data have been deposited in GEO with accession number GSE106588.

\section{Additional files}

Additional file 1: Figure S1. Biogenesis pathway and subcellular localization of miRs. miRs are mainly transcribed by RNA pol II, while a cluster of miRs flanked by Alu repeats on chromosome 19 (C19MC) are transcribed by RNA pol III [136], into pri-miRs (>1 kb long) with a hairpin structure [137]. Pri-miRs are recognized by Drosha, a class 2 RNAase III, and an RNA binding protein (RBP) called DGCR8/Pasha. Drosha cleaves the $5^{\prime}$ and $3^{\prime}$ arm of the hairpin releasing pre-miRs ( $70 \mathrm{bp}$ long). The latter are exported through the nuclear pores into the cytoplasm by Exportin 5 in association with Ran-GTP. In the cytoplasm processing of pre-miRs is mediated by Dicer, a class 3 RNase III, which along with various RBPs, including TRBP, stabilize Dicer. Dicer-TRBP complex liberates small RNA duplexes that are loaded onto Argonaute protein members (Ago1-4) forming effector complexes called pre-RISCs. Pre-RISCs remove the passenger miRs strand generating the mature form of RISCs encompassing single strand miRs ( 22 nucleotides long each). The functional strand of miRs loaded on Ago1-4 guides RISCs to silence target mRNAs in the cytoplasm (C) through translational repression, mRNA cleavage and deadenylation [138]. Additionally, miRs may translocate into: a) the nucleus (N) [16], regulating the biogenesis of coding and non-coding RNAs (active RISC complexes are present in the nucleus (nRISC) having a distinct composition from cytoplasmic RISC (CRISC) [139]) and b) the mitochondria (M) bound to Ago2 at pre-RISC or mature RISC complex (mRISC) [17], regulating the 
translation of the mRNAs produced by mitochondrial genome which, in turn, modulate mitochondrial homeostasis [140]. Evidence also supports the presence of mitochondrial miRs encoded by mitochondrial genome [18]. A substantial fraction of miRs may also exist in the cytoplasm in an Ago-free form [141]. Notably, apart from DGCR8 and TRBP, different RBPs recognize distinct miR precursors regulating miR biogenesis [142]. (PDF $1025 \mathrm{~kb}$ )

Additional file 2: Table S1. Subcellular localization of miRs. (XLSX $12 \mathrm{~kb}$ )

Additional file 3: Table S2. Studies on miR expression in senescent cells. (XLSX $20 \mathrm{~kb}$ )

Additional file 4: Table S3. Genes triggering oncogene-induced senescence. (XLSX $12 \mathrm{~kb}$ )

Additional file 5: Figure S2. RB phosphorylation in HBEC CDC6 Tet-ON system. Immunoblot analysis of total and phosphorylated RB levels. CDK4 over-expression in HBEC results in continuous phorsphorylation of RB protein, while induction of $C D C 6$ increased $\mathrm{p}-\mathrm{RB}$ due to transcriptional down-regulation of p16 [63]. Actin serves as loading control. (PDF $21 \mathrm{~kb}$ )

Additional file 6: Figure S3. CDC6 binding onto the promoters of CDH1 and INK4/ARF loci of HBEC CDC6 Tet-ON system leading to transcriptional down-regulation. a) Chromatin immunoprecipitation (ChIP) assay showed that MYC-tagged CDC6 is bound on both the regulatory domain (RD) of INK4/ARF locus and the Epal element of $\mathrm{CDH}$ 1, when induced. b) RD of INK4 locus is enriched in DNA extracted from both anti-CDC6 (endogenous and exogenous) and anti-MYC-tag (exogenous) IPs in HBEC CDC6 over-expressing cells normalized to input and INK4b intron (RNA Pol II-IP serves as a negative control confirming transcriptional down-regulation). c) ChIP samples run on a SDS-PAGE gel revealed that CDC6 is accessible and immunoprecipitated by both CDC6 and MYC-tag antibodies with the protocol followed. (PDF $146 \mathrm{~kb}$ )

Additional file 7: Figure S4. Morphological features of HBEC CDC6 Tet-ON. a) Inverted-phase contrast photographs (Scale bar: 25 um) and bi) GL13 staining showed the dominance of senescent, flattened and multinucleated cells upon 6-day CDC6-induction; features that were substituted by a spindle morphology in the "escaped" cells. Traces of GL13 staining in the early "escaped" cells (indicated by arrows) prove their origin from senescent cells. bii) Sa- $\beta$-Gal activity correlates with GL13 staining. (Scale bar: $15 \mu \mathrm{m}$ ). (PDF $689 \mathrm{~kb})$

Additional file 8: Figure S5. Comparative Inverted DAPI Banding karyotyping of 20 metaphase spreads from the OFF (on the left) and the "escaped" (on the right) cells. Arrows indicate random chromosome rearrangements (chromosomal instability). The rates of random structural chromosome rearrangements were found 3.5-times more pronounced in the "escaped" cells. (PDF 554 kb)

Additional file 9: Figure S6. Schematic presentation of an R loop. R loops are three-stranded nucleic acid structure. Factors (upper left corner) that promote R loops are indicated as well as the differential cellular effects (bottom) stemming from their formation. (PDF $557 \mathrm{~kb}$ )

Additional file 10: Figure S7. Bedgraphs of indicative genes showing the specificity of RNAseq analysis. RNAseq data from two biological replicates is depicted. (PDF $47 \mathrm{~kb}$ )

Additional file 11: Table S4. A Upregulated genes in induced (ON) HBEC CDC6 Tet-ON cells. b Down regulated genes in induced (ON) HBEC CDC6 Tet-ON cells. c Upregulated genes in ESCAPED (ESC) HBEC CDC6 Tet-ON cells. d: Down regulated genes in ESCAPED (ESC) HBEC CDC6 Tet-ON cells. (XLSX $630 \mathrm{~kb})$

Additional file 12: Figure S8. Enrichment plots a-b) of the "Cell cycle mitotic" and c-d) of the "DNA replication" gene-sets. Cells entering senescence (3-day induced) showed a significant (Bonferroni-adjusted $p$ value $<0.001$ ) down-regulation of cell-cycle and DNA replication pathways in comparison to control ones. These changes were reversed with a significant up-regulation (Bonferroni-adjusted $p$ value $<0.001$ ) of both sets when the cells escaped from senescence. (PDF $528 \mathrm{~kb}$ )

Additional file 13: Table S5. A MiR expression differences between induced (3d ON) and non-induced (OFF) HBEC CDC6 Tet-ON cells. FWER - Bonferroni-adjusted $p$ value. $b$ MiR expression differences between induced (6d ON) and non-induced (OFF) HBEC CDC6 Tet-ON cells.
FWER - Bonferroni-adjusted $p$ value. c MiR expression differences between "escaped" (ESC) and non-induced (OFF) HBEC CDC6 Tet-ON cells. FWER - Bonferroni-adjusted $p$ value. d MiR expression differences between 3-day induced (3d ON) and 6-day induced (6d ON) HBEC CDC6 Tet-ON cells. FWER - Bonferroni-adjusted $p$ value. e MiR expression differences between "escaped" (ESC) and induced (3d ON) HBEC CDC6 Tet-ON cells. FWER - Bonferroni-adjusted $p$ value. $f$ MiR expression differences between "escaped" (ESC) and induced ( $6 \mathrm{~d}$ ON) HBEC CDC6 Tet-ON cells. FWER - Bonferroni-adjusted p value. (XLSX $134 \mathrm{~kb}$ )

Additional file 14: Figure S9. miR-34c expression analysis in OFF, ON and "ESCAPED" HBEC CDC6 Tet-ON cells utilizing: a) qRT-PCR and b) miRseq analysis, tpm (transcripts per million). (PDF $25 \mathrm{~kb}$ )

Additional file 15: Table S6. Potential miR signature of senescence according to miRseq analysis of HBEC CDC6 Tet-ON system and existing literature. (XLSX $9 \mathrm{~kb}$ )

Additional file 16: Table S7. MiR enrichment analysis showing pathways, Gene Ontology terms and Target genes significantly linked to differentially-expressed miRs between respective groups.Fields marked out in bold represent pathways present in at least two pairwise comparisons. (XLSX $20 \mathrm{~kb}$ )

Additional file 17: Supplementary methods. (see also Additional file 18: Figure S10). (DOC $91 \mathrm{~kb})$

Additional file 18: Figure S10. Flowchart of the protocol employed to co-detect in situ gene coding (protein) and non-coding (miR) products during OIS in the HBECs CDC6 Tet-ON system. See also Additional file 17. (PDF $116 \mathrm{~kb}$ )

Additional file 19: Figure S11. Hybridization probes incorporating nucleotide analogs based on the LNA technology. (PDF $144 \mathrm{~kb}$ )

Additional file 20: Figure S12. Detection of U6 SnRNA and scramble-miR miRNACURY control double-DIG labeled probes employing TSA plus Fluorescein system. Scale bar: $30 \mu \mathrm{m}$. (PDF $74 \mathrm{~kb}$ )

\section{Abbreviations}

5'-EU: 5'-ethynyl uridine; BrdU: Bromodeoxyuridine; CDC6: Cell division cycle 6; CDK4: Cyclin-dependent kinase 4; CFS: Common fragile sites; ChIP: Chromatin immunoprecipitation; DDR: DNA damage response; DGCR8: DiGeorgi critical region 8; DIG: Double-digoxigenin; DSB: Double strand break; ECEE: Epithelial cancer evolution experiment; EDC: 1-ethyl-3(3-dimethylaminopropyl) carbodiimide; EMT: Epithelial to mesenchymal transition; FACS: Flow cytometric analysis; FISH: Fluorescence in situ hybridization; GO: Gene ontology; HBEC: Human bronchial epithelial cells; hTERT: Human telomerase reverse transcriptase; IF: Immunofluorescence; ISH: in situ hybridization; LNA: Locked nucleic acid; M-FISH/SKY: Multicolour fluorescence in situ hybridization/spectral karyotypicing; miR: Micro-RNA; ncRNA: Non-coding RNA; OIS: Oncogene-induced senescence; Pasha: Partner of Drosha; PO: Propylene oxide; pre-miR: Precursor micro-RNA; pri-miR: Primary micro-RNA; qRT-PCR: Quantitative reverse-transcription polymerase chain reaction; RBP: RNA binding protein; RD: Regulatory domain; rDNA: Ribosomal DNA; RISC: RNA-induced silencing complex; RNA pol: RNA polymerase; RNAseq: RNA sequencing; RS: Replicative senescence; RT: Room temperature; SAHF: Senescence associated heterochromatin foci; SASP: Senescence associated secretory phenotype; SA- $\beta$-Gal: Senescence-associated $\beta$-galactosidase; SIPS: Stress-induced premature senescence; snRNA: Small nuclear RNA; sSDNA: Single-stranded; TRBP: TAR RNA-binding protein; TSA: Tyramide signal amplification; UBF: Upstream binding factor

\section{Acknowledgements}

We would like to thank Professor Niels Ødum for helpful discussions, Dr. A. Kotsinas for his valuable support to this work, Dr. S. Havaki for help with the electron microscopy analysis, Dr. G. Panagopoulos for the RNAseq and miRseq analysis, as well as Dr. I. Kloukina and Prof. Capetanaki of the Center of Basic Research at Biomedical Research Foundation of Academy of Athens (BRFAA), for the use of Transmission Electron Microscope. We also thank C. Kotsi and I. Svolaki for technical support. 


\section{Funding}

V.G.G and E.-S.K. received a National Scholarships Foundation-Siemens Aristeia Fellowship. C.P.Z. was financially supported from the European Union's Horizon 2020 research and innovation programme under the Marie Sklodowska-Curie grant agreement No. 722729. Financial support was also provided by SARG-NKUA code 70/3/12128 and 70/3/8916. TK and NØ were supported by research funding from the Danish Research Council, the Danish Cancer Society and the Novo Nordic Foundation while TK was further supported by a Sapera Aude Talent Grant (DFF-4092-00122) from the Danish Council for Independent Research.

\section{Availability of data and materials}

RNAseq data have been deposited in the Short Read Archive (SRA) under the accession codes PRJNA388146. MiRseq data have been deposited in GEO with accession number GSE106588. The GEO submission is published and available using https://www.ncbi.n/m.nih.gov/geo/query/ acc.cgi?acc=GSE106588.

\section{Authors' contributions}

E-SK developed the cellular system. ISP, SVR, EP, CPZ and TK participated in cell culture and conducted in situ techniques (FISH, senescence staining, double and triple detections). E-SK, IM, SG and IPT performed cell culture manipulations, cell growth assays, SA- $\beta$-Gal assay, immunoblots, IF analysis, $\mathrm{RNA} / \mathrm{miR}$ isolation, qRT-PCR, comet assay, electron microscopy, FACS analysis and ChIP. AP, KS, WF and CD performed RNAseq and miRseq along with the analysis of the results. FMR, MC and SG performed the cytogenetic analysis. D-EP and EON were responsible for the design, experimental procedure and interpretation of the 5'-EU assay and provision of specialized reagents. IM, CPZ and TK conducted literature search and Table preparation. E-SK and ISP performed figure preparation. AGE and JB assisted in data analysis. VGG designed the experiments, supervised the project and wrote the manuscript. All authors read and approved the final manuscript.

\section{Ethics approval and consent to participate}

Not applicable

\section{Consent for publication}

Not applicable

\section{Competing interests}

The authors declare that they have no competing interests.

\section{Author details}

'Molecular Carcinogenesis Group, Department of Histology and Embryology, School of Medicine, National \& Kapodistrian University of Athens, 75 Mikras Asias St, GR-11527 Athens, Greece. ²Department of Immunology and Microbiology, University of Copenhagen, Blegdamsvej 3c, DK-2200 Copenhagen, Denmark. ${ }^{3}$ Department of Biostatistics and Translational Medicine, Medical University of Lodz, 15 Mazowiecka St. 92-215, Lodz, Poland. ${ }^{4}$ Biomedical Research Foundation of the Academy of Athens, 4 Soranou Ephessiou St, GR-11527 Athens, Greece. ${ }^{5}$ Department of Cell Biology and Biophysics, Faculty of Biology, National \& Kapodistrian University of Athens, GR-15784 Athens, Greece. ${ }^{6}$ CRUK/MRC Institute for Radiation Oncology, Department of Oncology, University of Oxford, Oxford OX3 7DQ, UK. ${ }^{7}$ Department of Biology, School of Medicine, National \& Kapodistrian University of Athens, 75 Mikras Asias St, GR-11527 Athens, Greece. ${ }^{8}$ Institute of Molecular Biology and Biotechnology, Foundation for Research \& Technology-Hellas, GR-70013 Heraklion, Crete, Greece. ${ }^{9}$ Department of Radiation Oncology, Dana-Farber Cancer Institute, 450 Brookline Ave, Boston, MA 02215, USA. ${ }^{10}$ Harvard Medical School, 25 Shattuck St, Boston, MA 02115, USA. ${ }^{11}$ Genome Integrity Unit, Danish Cancer Society Research Centre, Strandboulevarden 49, DK-2100 Copenhagen, Denmark. ${ }^{12}$ Institute of Molecular and Translational Medicine, Faculty of Medicine and Dentistry, Palacky University, Hněvotínská, 1333/5, 77900 Olomouc, Czech Republic. ${ }^{13}$ Department of Medical Biochemistry and Biophysics, Karolinska Institute, Science for Life Laboratory, Division of Translational Medicine and Chemical Biology, SE-171 77 Stockholm, Sweden. ${ }^{14}$ Faculty of Biology, Medicine and Health, University of Manchester, Manchester Academic Health Science Centre, Wilmslow Road, Manchester M20 4QL, UK.
Received: 12 July 2017 Accepted: 11 December 2017

Published online: 10 January 2018

\section{References}

1. Braunschweig $U$, et al. Dynamic integration of splicing within gene regulatory pathways. Cell. 2013;152(6):1252-69.

2. Brosius J. The persistent contributions of RNA to eukaryotic gen(om)e architecture and cellular function. Cold Spring Harb Perspect Biol. 2014; 6(12):a016089.

3. Jiao $Y$, Torquato $S$. Emergent behaviors from a cellular automaton model for invasive tumor growth in heterogeneous microenvironments. PLoS Comput Biol. 2011;7(12):e1002314.

4. Hanahan D, Weinberg RA. The hallmarks of cancer. Cell. 2000:100(1):57-70.

5. Egeblad M, Nakasone ES, Werb Z. Tumors as organs: complex tissues that interface with the entire organism. Dev Cell. 2010;18(6):884-901.

6. Flynt AS, Lai EC. Biological principles of microRNA-mediated regulation: shared themes amid diversity. Nat Rev Genet. 2008;9(11):831-42.

7. Sun K, Lai EC. Adult-specific functions of animal microRNAs. Nat Rev Genet. 2013;14(8):535-48.

8. Mendell JT, Olson EN. MicroRNAs in stress signaling and human disease Cell. 2012;148(6):1172-87.

9. Eguchi T, Kuboki T. Cellular reprogramming using defined factors and MicroRNAs. Stem Cells Int. 2016;2016:7530942.

10. Ambros V. The functions of animal microRNAs. Nature. 2004:431(7006):350-5.

11. Gruber AJ, Zavolan M. Modulation of epigenetic regulators and cell fate decisions by miRNAs. Epigenomics. 2013;5(6):671-83.

12. Erhard F, et al. Widespread context dependency of microRNA-mediated regulation. Genome Res. 2014;24(6):906-19.

13. Lee RC, Feinbaum RL, Ambros V. The C. Elegans heterochronic gene lin-4 encodes small RNAs with antisense complementarity to lin-14. Cell. 1993;75(5):843-54.

14. Olena AF, Patton JG. Genomic organization of microRNAs. J Cell Physiol. 2010:222(3):540-5

15. Bartel DP. MicroRNAs: genomics, biogenesis, mechanism, and function. Cell. 2004;116(2):281-97.

16. Rasko JE, Wong JJ. Nuclear microRNAs in normal hemopoiesis and cancer. J Hematol Oncol. 2017;10(1):8.

17. Srinivasan H, Das S. Mitochondrial miRNA (MitomiR): a new player in cardiovascular health. Can J Physiol Pharmacol. 2015;93(10):855-61.

18. Barrey $E$, et al. Pre-microRNA and mature microRNA in human mitochondria. PLoS One. 2011;6(5):e20220.

19. Williams J, et al. Are microRNAs true sensors of ageing and cellular senescence? Ageing Res Rev. 2017;35:350-63.

20. Abdelmohsen K, Gorospe M. Noncoding RNA control of cellular senescence. Wiley Interdiscip Rev RNA. 2015;6(6):615-29.

21. Bischof O, Martinez-Zamudio RI. MicroRNAs and IncRNAs in senescence: a re-view. IUBMB Life. 2015;67(4):255-67.

22. Olivieri $F$, et al. DNA damage response (DDR) and senescence: shuttled inflamma-miRNAs on the stage of inflamm-aging. Oncotarget. 2015:6(34):35509-21.

23. Campisi J, Robert L. Cell senescence: role in aging and age-related diseases. Interdiscip Top Gerontol. 2014;39:45-61.

24. Munoz-Espin D, Serrano M. Cellular senescence: from physiology to pathology. Nat Rev Mol Cell Biol. 2014;15(7):482-96.

25. Giaimo S, d'Adda F. Di Fagagna, is cellular senescence an example of antagonistic pleiotropy? Aging Cell. 2012;11(3):378-83.

26. Gorgoulis VG, Halazonetis TD. Oncogene-induced senescence: the bright and dark side of the response. Curr Opin Cell Biol. 2010;22(6):816-27.

27. Sikora $\mathrm{E}$, et al. Impact of cellular senescence signature on ageing research. Ageing Res Rev. 2011;10(1):146-52.

28. Braig $M$, et al. Oncogene-induced senescence as an initial barrier in lymphoma development. Nature. 2005;436(7051):660-5.

29. Chen Z, et al. Crucial role of p53-dependent cellular senescence in suppression of Pten-deficient tumorigenesis. Nature. 2005;436(7051):725-30.

30. Collado $\mathrm{M}$, et al. Tumour biology: senescence in premalignant tumours. Nature. 2005;436(7051):642.

31. Lazzerini Denchi E, et al. Deregulated E2F activity induces hyperplasia and senescence-like features in the mouse pituitary gland. Mol Cell Biol. 2005; 25(7):2660-72

32. Michaloglou C, et al. BRAFE600-associated senescence-like cell cycle arrest of human naevi. Nature. 2005;436(7051):720-4. 
33. Bartkova J, et al. Oncogene-induced senescence is part of the tumorigenesis barrier imposed by DNA damage checkpoints. Nature. 2006:444(71 19):633-7.

34. Di Micco R, et al. Oncogene-induced senescence is a DNA damage response triggered by DNA hyper-replication. Nature. 2006;444(71 19):638-42.

35. Serrano $M$, et al. Oncogenic ras provokes premature cell senescence associated with accumulation of p53 and p16INK4a. Cell. 1997;88(5):593-602.

36. Halazonetis TD, Gorgoulis VG, Bartek J. An oncogene-induced DNA damage model for cancer development. Science. 2008;319(5868):1352-5.

37. Geraghty RJ, et al. Guidelines for the use of cell lines in biomedical research Br J Cancer. 2014;111(6):1021-46.

38. Dickson MA, et al. Human keratinocytes that express hTERT and also bypass a p16(INK4a)-enforced mechanism that limits life span become immortal yet retain normal growth and differentiation characteristics. Mol Cell Biol. 2000;20(4):1436-47.

39. Kiyono $\mathrm{T}$, et al. Both Rb/p16INK4a inactivation and telomerase activity are required to immortalize human epithelial cells. Nature. 1998;396(6706):84-8.

40. Dimri GP, et al. A biomarker that identifies senescent human cells in culture and in aging skin in vivo. Proc Natl Acad Sci U S A. 1995;92(20):9363-7.

41. AbouHaidar MG, Ivanov IG. Non-enzymatic RNA hydrolysis promoted by the combined catalytic activity of buffers and magnesium ions. Z Naturforsch C. 1999:54(7-8):542-8.

42. Chen SJ. RNA folding: conformational statistics, folding kinetics, and ion electrostatics. Annu Rev Biophys. 2008;37:197-214.

43. Koculi $E$, et al. Charge density of divalent metal cations determines RNA stability. J Am Chem Soc. 2007;129(9):2676-82.

44. Draper DE. RNA folding: thermodynamic and molecular descriptions of the roles of ions. Biophys J. 2008;95(12):5489-95.

45. Hertweck M, Mueller MW. Mapping divalent metal ion binding sites in a group II intron by $\mathrm{Mn}(2+)$ - and $\mathrm{Zn}(2+)$-induced site-specific RNA cleavage. Eur J Biochem. 2001;268(17):4610-20.

46. $X i$ Y, et al. Systematic analysis of microRNA expression of RNA extracted from fresh frozen and formalin-fixed paraffin-embedded samples. RNA. 2007;13(10):1668-74.

47. Meng $W$, et al. Comparison of microRNA deep sequencing of matched formalin-fixed paraffin-embedded and fresh frozen cancer tissues. PLoS One. 2013;8(5):e64393

48. Jung $M$, et al. Robust microRNA stability in degraded RNA preparations from human tissue and cell samples. Clin Chem. 2010;56(6):998-1006.

49. Peskoe SB, et al. Differential long-term stability of microRNAs and RNU6B snRNA in 12-20 year old archived formalin-fixed paraffin-embedded specimens. BMC Cancer. 2017;17(1):32.

50. Rentoft $M$, et al. miRNA analysis of formalin-fixed squamous cell carcinomas of the tongue is affected by age of the samples. Int J Oncol. 2011;38(1):61-9.

51. Mall C, et al. Stability of miRNA in human urine supports its biomarker potential. Biomark Med. 2013;7(4):623-31.

52. Esser $C$, et al. Isolation of full-size mRNA from ethanol-fixed cells after cellular immunofluorescence staining and fluorescence-activated cell sorting (FACS). Cytometry. 1995;21(4):382-6.

53. Evangelou $\mathrm{K}$, et al. Robust, universal biomarker assay to detect senescent cells in biological specimens. Aging Cell. 2017;16(1):192-7.

54. Georgakopoulou EA, et al. Specific lipofuscin staining as a novel biomarker to detect replicative and stress-induced senescence. A method applicable in cryo-preserved and archival tissues. Aging (Albany NY). 2013;5(1):37-50.

55. Gorgoulis VG, et al. Activation of the DNA damage checkpoint and genomic instability in human precancerous lesions. Nature. 2005;434(7035):907-13.

56. Georgakilas AG, et al. Are common fragile sites merely structural domains or highly organized "functional" units susceptible to oncogenic stress? Cell Mol Life Sci. 2014;71(23):4519-44.

57. Ramirez RD, et al. Immortalization of human bronchial epithelial cells in the absence of viral oncoproteins. Cancer Res. 2004;64(24):9027-34

58. Evangelou $\mathrm{K}$, et al. The DNA damage checkpoint precedes activation of ARF in response to escalating oncogenic stress during tumorigenesis. Cell Death Differ. 2013;20(11):1485-97.

59. Velimezi $G$, et al. Functional interplay between the DNA-damage-response kinase ATM and ARF tumour suppressor protein in human cancer. Nat Cell Biol. 2013;15(8):967-77

60. Liontos $\mathrm{M}$, et al. Deregulated overexpression of hCdt1 and hCdc6 promotes malignant behavior. Cancer Res. 2007;67(22):10899-909.

61. Karakaidos $P$, et al. Overexpression of the replication licensing regulators hCdt1 and hCdc6 characterizes a subset of non-small-cell lung carcinomas: synergistic effect with mutant p53 on tumor growth and chromosomal instability-evidence of E2F-1 transcriptional control over hCdt1. Am J Pathol. 2004;165(4):1351-65.

62. Petrakis TG, et al. Exploring and exploiting the systemic effects of deregulated replication licensing. Semin Cancer Biol. 2016;37-38:3-15.

63. Sideridou $\mathrm{M}_{\text {, et al. }} \mathrm{Cdc} 6$ expression represses E-cadherin transcription and activates adjacent replication origins. J Cell Biol. 2011;195(7):1123-40.

64. Galanos P, et al. Chronic p53-independent p21 expression causes genomic instability by deregulating replication licensing. Nat Cell Biol. 2016;18(7):777-89.

65. Petrakis TG, Vougas K, Gorgoulis VG. Cdc6: a multi-functional molecular switch with critical role in carcinogenesis. Transcription. 2012;3(3):124-9.

66. Huang $S$, et al. DNA replication initiator $C d c 6$ also regulates ribosomal DNA transcription initiation. J Cell Sci. 2016:129(7):1429-40.

67. Georgakopoulou E, et al. Apoptosis or senescence? Which exit route do epithelial cells and fibroblasts preferentially follow? Mech Ageing Dev. 2016;156:17-24

68. Niimi S, et al. Cdc6 protein obstructs apoptosome assembly and consequent cell death by forming stable complexes with activated Apaf-1 molecules. J Biol Chem. 2012;287(22):18573-83.

69. Narita $\mathrm{M}$, et al. Rb-mediated heterochromatin formation and silencing of E2F target genes during cellular senescence. Cell. 2003;113(6):703-16.

70. Nieto MA, et al. Emt: 2016. Cell. 2016;166(1):21-45.

71. Thiery JP, et al. Epithelial-mesenchymal transitions in development and disease. Cell. 2009;139(5):871-90.

72. Liontos $\mathrm{M}$, et al. Modulation of the E2F1-driven cancer cell fate by the DNA damage response machinery and potential novel E2F1 targets in osteosarcomas. Am J Pathol. 2009;175(1):376-91.

73. Negrini S, Gorgoulis VG, Halazonetis TD. Genomic instability-an evolving hallmark of cancer. Nat Rev Mol Cell Biol. 2010;11(3):220-8.

74. d'Adda di Fagagna F. Living on a break: cellular senescence as a DNA-damage response. Nat Rev Cancer. 2008;8(7):512-22.

75. Walter D, et al. SCF(Cyclin F)-dependent degradation of CDC6 suppresses DNA re-replication. Nat Commun. 2016;7:10530.

76. Heng HH, et al. Chromosomal instability (CIN): what it is and why it is crucial to cancer evolution. Cancer Metastasis Rev. 2013;32(3-4):325-40.

77. Rangel N, Forero-Castro M, Rondon-Lagos M. New insights in the cytogenetic practice: Karyotypic chaos, non-Clonal chromosomal alterations and chromosomal instability in human cancer and therapy response. Genes (Basel). 2017;8(6):155

78. Mrasek K, et al. Global screening and extended nomenclature for 230 aphidicolin-inducible fragile sites, including 61 yet unreported ones. Int J Oncol. 2010;36(4):929-40.

79. Tan DS, et al. Intertumor heterogeneity of non-small-cell lung carcinomas revealed by multiplexed mutation profiling and integrative genomics. Int J Cancer. 2014:135(5):1092-100.

80. Kang J. Genomic alterations on 8p21-p23 are the most frequent genetic events in stage I squamous cell carcinoma of the lung. Exp Ther Med. 2015;9(2):345-50.

81. Jimenez-Sousa MA, et al. Myelodysplastic syndrome with isochromosome $5 p$ and trisomy 8 after treatment of a multiple myeloma. Cancer Genet Cytogenet. 2010;203(2):345-7.

82. Hoglund $M$, et al. Statistical dissection of cytogenetic patterns in lung cancer reveals multiple modes of karyotypic evolution independent of histological classification. Cancer Genet Cytogenet. 2004;154(2):99-109.

83. Skourti-Stathaki K, Proudfoot NJ. A double-edged sword: R loops as threats to genome integrity and powerful regulators of gene expression. Genes Dev. 2014:28(13):1384-96.

84. Roy D, Lieber MR. G clustering is important for the initiation of transcription-induced R-loops in vitro, whereas high $\mathrm{G}$ density without clustering is sufficient thereafter. Mol Cell Biol. 2009;29(11):3124-33.

85. Roy D, et al. Competition between the RNA transcript and the nontemplate DNA strand during R-loop formation in vitro: a nick can serve as a strong R-loop initiation site. Mol Cell Biol. 2010;30(1):146-59.

86. Xu B, Clayton DA. RNA-DNA hybrid formation at the human mitochondrial heavy-strand origin ceases at replication start sites: an implication for RNA-DNA hybrids serving as primers. EMBO J. 1996:15(12):3135-43.

87. Baker TA, Kornberg A. Transcriptional activation of initiation of replication from the E. Coli chromosomal origin: an RNA-DNA hybrid near oriC. Cell. 1988;55(1):113-23.

88. Carles-Kinch K, Kreuzer KN. RNA-DNA hybrid formation at a bacteriophage T4 replication origin. J Mol Biol. 1997;266(5):915-26. 
89. Masukata $\mathrm{H}$, Tomizawa J. Effects of point mutations on formation and structure of the RNA primer for ColE1 DNA replication. Cell. 1984;36(2):513-22.

90. Masukata $\mathrm{H}$, Tomizawa J. A mechanism of formation of a persistent hybrid between elongating RNA and template DNA. Cell. 1990;62(2):331-8.

91. Chan YA, et al. Genome-wide profiling of yeast DNA:RNA hybrid prone sites with DRIP-chip. PLoS Genet. 2014;10(4):e1004288.

92. El Hage A, et al. Genome-wide distribution of RNA-DNA hybrids identifies RNase $\mathrm{H}$ targets in tRNA genes, retrotransposons and mitochondria. PLoS Genet. 2014;10(10):e1004716.

93. Starokadomskyy $\mathrm{P}$, et al. DNA polymerase-alpha regulates the activation of type I interferons through cytosolic RNA:DNA synthesis. Nat Immunol. 2016;17(5):495-504.

94. Lima WF, et al. Viable RNaseH1 knockout mice show RNaseH1 is essential for R loop processing, mitochondrial and liver function. Nucleic Acids Res. 2016:44(11):5299-312.

95. Chiolo I, et al. Double-strand breaks in heterochromatin move outside of a dynamic HP1a domain to complete recombinational repair. Cell. 2011;144(5):732-44.

96. van Sluis M, McStay B. A localized nucleolar DNA damage response facilitates recruitment of the homology-directed repair machinery independent of cell cycle stage. Genes Dev. 2015;29(11):1151-63.

97. Grob A, Colleran C, McStay B. Construction of synthetic nucleoli in human cells reveals how a major functional nuclear domain is formed and propagated through cell division. Genes Dev. 2014;28(3):220-30.

98. Santos-Pereira JM, Aguilera A. R loops: new modulators of genome dynamics and function. Nat Rev Genet. 2015;16(10):583-97.

99. Boque-Sastre R, et al. Head-to-head antisense transcription and R-loop formation promotes transcriptional activation. Proc Natl Acad Sci U S A. 2015;112(18):5785-90.

100. Hanahan D, Weinberg RA. Hallmarks of cancer: the next generation. Cell. 2011;144(5):646-74.

101. Solimini NL, Luo J, Elledge SJ. Non-oncogene addiction and the stress phenotype of cancer cells. Cell. 2007;130(6):986-8.

102. Zaravinos A. The regulatory role of MicroRNAs in EMT and cancer. J Oncol. 2015;2015:865816.

103. Siemens $H$, et al. miR-34 and SNAIL form a double-negative feedback loop to regulate epithelial-mesenchymal transitions. Cell Cycle. 2011;10(24):4256-71.

104. He L, et al. microRNAs join the p53 network-another piece in the tumour-suppression puzzle. Nat Rev Cancer. 2007;7(11):819-22.

105. Disayabutr $\mathrm{S}$, et al. miR-34 miRNAs regulate cellular senescence in type II alveolar epithelial cells of patients with idiopathic pulmonary fibrosis. PLoS One. 2016;11(6):e0158367.

106. Bartek J, Lukas J. DNA damage checkpoints: from initiation to recovery or adaptation. Curr Opin Cell Biol. 2007;19(2):238-45.

107. Rappold l, et al. Tumor suppressor p53 binding protein 1 (53BP1) is involved in DNA damage-signaling pathways. J Cell Biol. 2001;153(3):613-20.

108. Bartkova J, et al. DNA damage response as a candidate anti-cancer barrier in early human tumorigenesis. Nature. 2005;434(7035):864-70.

109. Obernosterer G, Martinez J, Alenius M. Locked nucleic acid-based in situ detection of microRNAs in mouse tissue sections. Nat Protoc. 2007;2(6):1508-14.

110. Sempere LF, Korc M. A method for conducting highly sensitive microRNA in situ hybridization and immunohistochemical analysis in pancreatic cancer. Methods Mol Biol. 2013;980:43-59.

111. Nielsen BS, Holmstrom K. Combined microRNA in situ hybridization and immunohistochemical detection of protein markers. Methods Mol Biol. 2013;986:353-65.

112. Vester B, Wengel J. LNA (locked nucleic acid): high-affinity targeting of complementary RNA and DNA. Biochemistry. 2004;43(42):13233-41.

113. Silahtaroglu AN, et al. Detection of microRNAs in frozen tissue sections by fluorescence in situ hybridization using locked nucleic acid probes and tyramide signal amplification. Nat Protoc. 2007;2(10):2520-8.

114. de Planell-Saguer M, Rodicio MC, Mourelatos Z. Rapid in situ codetection of noncoding RNAs and proteins in cells and formalin-fixed paraffin-embedded tissue sections without protease treatment. Nat Protoc. 2010;5(6):1061-73.

115. Kwon S. Single-molecule fluorescence in situ hybridization: quantitative imaging of single RNA molecules. BMB Rep. 2013;46(2):65-72.

116. Pena JT, et al. miRNA in situ hybridization in formaldehyde and EDC-fixed tissues. Nat Methods. 2009;6(2):139-41.

117. Le Magnen C, Dutta A, Abate-Shen C. Optimizing mouse models for precision cancer prevention. Nat Rev Cancer. 2016;16(3):187-96.
118. Rangarajan A, et al. Species- and cell type-specific requirements for cellular transformation. Cancer Cell. 2004;6(2):171-83.

119. Kamijo T, et al. Loss of the ARF tumor suppressor reverses premature replicative arrest but not radiation hypersensitivity arising from disabled atm function. Cancer Res. 1999;59(10):2464-9.

120. Kamijo T, et al. Tumor suppression at the mouse INK4a locus mediated by the alternative reading frame product p19ARF. Cell. 1997;91(5):649-59.

121. Efeyan A, et al. Limited role of murine ATM in oncogene-induced senescence and p53-dependent tumor suppression. PLoS One. 2009;4(5):e5475.

122. Balmain A, Harris CC. Carcinogenesis in mouse and human cells: parallels and paradoxes. Carcinogenesis. 2000;21(3):371-7.

123. McStay B, Grummt I. The epigenetics of rRNA genes: from molecular to chromosome biology. Annu Rev Cell Dev Biol. 2008;24:131-57.

124. Murray JM, Stiff T, Jeggo PA. DNA double-strand break repair within heterochromatic regions. Biochem Soc Trans. 2012;40(1):173-8.

125. Nakane PK. Simultaneous localization of multiple tissue antigens using the peroxidase-labeled antibody method: a study on pituitary glands of the rat. J Histochem Cytochem. 1968;16(9):557-60.

126. Abbadie C, Pluquet O, Pourtier A. Epithelial cell senescence: an adaptive response to pre-carcinogenic stresses? Cell Mol Life Sci. 2017;74(24):4471-509.

127. Debacq-Chainiaux F, et al. Protocols to detect senescence-associated beta-galactosidase (SA-betagal) activity, a biomarker of senescent cells in culture and in vivo. Nat Protoc. 2009:4(12):1798-806.

128. Kim D, et al. TopHat2: accurate alignment of transcriptomes in the presence of insertions, deletions and gene fusions. Genome Biol. 2013;14(4):R36.

129. Li H, et al. The sequence alignment/map format and SAMtools. Bioinformatics. 2009;25(16):2078-9.

130. Anders S, Pyl PT, Huber W. HTSeq-a python framework to work with high-throughput sequencing data. Bioinformatics. 2015;31(2):166-9.

131. Anders S, Huber W. Differential expression analysis for sequence count data. Genome Biol. 2010;11(10):R106.

132. Chen S, et al. AfterQC: automatic filtering, trimming, error removing and quality control for fastq data. BMC Bioinformatics. 2017;18(Suppl 3):80.

133. Huang da W, Sherman BT, Lempicki RA. Systematic and integrative analysis of large gene lists using DAVID bioinformatics resources. Nat Protoc. 2009:4(1):44-57.

134. Subramanian A, et al. Gene set enrichment analysis: a knowledge-based approach for interpreting genome-wide expression profiles. Proc Natl Acad Sci U S A. 2005;102(43):15545-50.

135. Backes $C$, et al. miEAA: microRNA enrichment analysis and annotation. Nucleic Acids Res. 2016:44(W1):W110-6.

136. Borchert GM, Lanier W, Davidson BL. RNA polymerase III transcribes human microRNAs. Nat Struct Mol Biol. 2006;13(12):1097-101.

137. Ha M, Kim VN. Regulation of microRNA biogenesis. Nat Rev Mol Cell Biol. 2014;15(8):509-24.

138. Winter J, et al. Many roads to maturity: microRNA biogenesis pathways and their regulation. Nat Cell Biol. 2009;11(3):228-34.

139. Roberts TC. The MicroRNA biology of the mammalian nucleus. Mol Ther Nucleic Acids. 2014:3:e188.

140. Baradan R, Hollander JM, Das S. Mitochondrial miRNAs in diabetes: just the tip of the iceberg. Can J Physiol Pharmacol. 2017;95:1156-62.

141. Makarova JA, et al. Intracellular and extracellular microRNA: an update on localization and biological role. Prog Histochem Cytochem. 2016;51(3-4):33-49.

142. Treiber $T$, et al. A compendium of RNA-binding proteins that regulate MicroRNA biogenesis. Mol Cell. 2017;66(2):270-84. e13

143. Sakellariou $D$, et al. Alternative lengthening of telomeres: recurrent cytogenetic aberrations and chromosome stability under extreme telomere dysfunction. Neoplasia. 2013;15(11):1301-13. 